\title{
A Comparison Study: The New Extended Shelf Life Isopropyl Ester PMR Technology versus The Traditional Methyl Ester PMR Approach
}

\author{
By
}

William B. Alston*

U.S.Army Research Laboratory (ARL), Vehicle Technology Directorate (VTD), Daniel A. Scheiman, QSS Group, Inc., and

Gloria S. Sivko, Ohio Aerospace Institute (OAI),

All three authors co-located at NASA-Glenn Research Center, Cleveland, Ohio 44135

\begin{abstract}
$\underline{\text { Abstract }}$
Polymerization of Monomeric Reactants (PMR) monomer solutions and carbon cloth prepregs of PMR II-50 and VCAP-75 were prepared using both the traditional limited shelf life methanol based PMR approach and a novel extended shelf life isopropanol based PMR approach. The methyl ester and isopropyl ester based PMR monomer solutions and PMR prepregs were aged for up to four years at freezer and room temperatures. The aging products formed were monitored using high pressure liquid chromatography (HPLC). The composite processing flow characteristics and volatile contents of the aged prepregs were also correlated versus room temperature storage time. Composite processing cycles were developed and six ply cloth laminates were fabricated with prepregs after various extended room temperature storage times. The composites were then evaluated for glass transition temperature $\left(\mathrm{T}_{\mathrm{g}}\right)$, thermal decomposition temperature $\left(\mathrm{T}_{\mathrm{d}}\right)$, initial flexural strength (FS) and modulus (FM), long term (1000 hours at $316^{\circ} \mathrm{C}$ ) thermal oxidative stability (TOS), and retention of FS and FM after 1000 hours aging at $316^{\circ} \mathrm{C}$. The results for each ester system were comparable. Freezer storage was found to prevent the formation of aging products for both ester systems. Room temperature storage of the novel isopropyl ester system increased PMR monomer solution and PMR prepreg shelf life by at least an order of magnitude while maintaining composite properties.
\end{abstract}

\section{Introduction}

PMR (Polymerization Monomer Reactants) type resins ${ }^{1-3}$ are currently employed in a variety of high technology applications. Their high temperature performance makes them excellent candidates for weight-saving applications in aircraft engines. VCAP- $75^{2}$ (vinyl endcap of 7874 formulated molecular weight (FMW), $\mathrm{n}=14$ ) and PMR II-50 ${ }^{3}$ (second generation PMR at $5047 \mathrm{FMW}, \mathrm{n}=9$ ) are two such promising polyimides undergoing commercialization. Both required the development of optimal processing parameters and quality control techniques. Even with optimal processing conditions an important factor affecting the quality of the polymer precursor solutions is their finite shelf life. ${ }^{4}$ Historically storage stability has been a problem associated with both first and second generation type PMR resin systems. ${ }^{5}$ PMR monomer solutions and PMR prepregs (monomer impregnated fiber or fabric) storage stability depends on temperature,

This is a preprint of an article submitted to a journal for publication. Since revisions may be made prior to formal publication, this version is made available with the understanding that it will not be cited or reproduced without the permission of the author. 
monomer reaction rates, concentrations, and reagent purity. Chemical reaction information about the PMR monomer solution and PMR prepreg stabilities can be obtained using a variety of analytical techniques.

One of the primary concerns about solution and prepreg storage is reactions between the monomers resulting in undesirable aging products. The polyimides in this study are thermoset polymers formed through thermally driven condensation reactions between diamines, carboxylic diacid diesters and monofunctional endcaps. However, the monomers can combine at much lower temperatures, even room temperature, to produce a variety of adducts or aging products. Potentially, the most detrimental aging products are the chain extending combinations between both monomers, which can cause a significant increase in melt viscosity. However the reactions between some PMR endcaps and one or both ends of a monomer, while not chain extending reactions, can occur at a much faster rate, thereby also significantly increase melt viscosity. Both of these product types will undesirably affect the composite processing and ultimately the finished laminate properties. Thus a study using aged prepregs and examining the resultant laminate properties should reveal any inherent undesired effects from the prepreg storage.

The overall objective of this study was to identify the PMR monomer solution and prepreg aging processes and to characterize their effects in traditional methyl ester PMR monomer solutions, prepregs and composites, followed by demonstrating the lack of these aging effects when using extended shelf life isopropyl ester PMR monomer solutions, prepregs and composites.

The approach used for this study involved the monitoring of methyl ester second generation type PMR monomer solutions and prepregs using high pressure liquid chromatography (HPLC) and rheology at various room temperature storage time intervals (from 0 to 6 months) to track the formation of aging products and subsequent viscosity changes. $^{6,7}$ The PMR prepreg materials were then processed into laminates at the same time intervals. ${ }^{8-10}$ The prepreg volatile contents and flow characteristics were correlated versus prepreg room temperature storage time. The quality of the laminates was evaluated using ultrasonic C-scans, thermal analysis, and mechanical testing. Long term oven aging to monitor the high temperature stability (via isothermal weight loss) was also done followed by mechanical testing of the oven aged laminates to determine the retention of composite mechanical strength. Differences noted in the aged prepregs and monomer solutions were then correlated with the changes in the laminate properties.

The alternative novel extended shelf life technology was then applied to the second generation type PMR polyimides. A similar characterization scheme as described above was performed for their solutions, prepregs and composites after up to fifty months of room temperature prepreg storage times. Some of these results were presented earlier. ${ }^{11}$ Solution solubility and stability studies of first generation type polyimides and their composite fabrication ${ }^{12}$ have also been previously reported. ${ }^{12-14}$ In those studies methyl ester and isopropyl ester solution shelf life ${ }^{12}$ was compared and the use of various cosolvents ${ }^{13-14}$ was also reported to extend PMR shelf life. All the second generation results (along with the methyl ester comparisons) are now presented in this overall comparison study.

The reason the extended shelf life technology provides longer shelf life is because it is based on the larger isopropyl ester groups compared to the methyl ester group. The isopropyl group is a bulkier and poorer leaving group and consequently should 
significantly slow the reactions between the PMR monomers in solutions and prepregs by slowing the rate determining step in the aging process. The mechanistic rate determining step in the aging process has been identified ${ }^{15}$ as anhydride reformation from the ester acid. This aging of methyl ester PMR monomer solutions and prepregs is significantly noticeable even during room temperature storage. The anhydride reformation rate determining step is always followed by rapid reaction of the anhydrides with amines, even at low temperatures, thus forming amide acids. Amide acids then may further react by rapidly dehydrating to form imides, even sometimes at room temperature. These imides are traditionally identified as the products of the PMR aging process. However, they are really only premature normal imidization products forming before the final composite processing. This narrows the traditional methyl ester PMR composite processing window. The lack of aging in the isopropyl ester PMR approach could lead to more consistent composite processability and composite properties due to the longer allowable working "out life" time during composite processing. The lack of aging also provides a bonus of increased allowable prepreg storage time and temperature compared to methyl ester shelf life conditions. By significantly slowing room temperature imidization with isopropyl esters, premature aging is prevented, thus potentially keeping the processing window at its maximum size.

\section{Experimental}

\subsection{PMR Monomer Synthesis}

Monomers and endcaps for VCAP-75 and PMR II-50 used to prepare the standards for the HPLC analysis and to prepare the PMR solutions and the prepregs are shown in Figure 1. A 50 weight percent solution of 6F diphthalic acid ester (HFDE) was prepared using 1,1,1,3,3,3-hexafluoroisopropylidine diphthalic anhydride (HFDA, Hoechst Chemical) by heating at reflux temperature with the appropriate alcohol for an additional 1-2 hours in methanol or isopropanol after dissolution of the HFDA (which took 1-2 hours in methanol or 5-6 hours in isopropanol. The para-aminostyrene (PAS, Polyscience), para-phenylenediamine (PPDA, DuPont) and methyl nadic acid ester (NE, Pharm-Eco Laboratories) were used as received. Nadic anhydride (NA, Hitco Lab) was heated at reflux temperature in isopropanol for 24 hours to generate the isopropyl nadic acid ester according to the patented procedure. ${ }^{16}$ The isopropyl nadic acid ester was isolated by filtration and recrystallized from $n$-hexane in $98 \%$ yield, melting point 89 $90^{\circ} \mathrm{C}$. A $1 \%$ impurity, the nadic diacid, was shown by HPLC. All compounds were run in the HPLC individually to check retention times and purity. Some of the aging products (mono and bisnadimides of PPDA, and mono, tri, and tetraesters of HFDA) were synthesized in house using the appropriate monomers and/or purified for use as HPLC standards to identify their retention times.

\subsection{PMR Monomer Solution and Prepreg Preparation}

The PMR solutions were prepared in a traditional manner. The monomers were mixed together in methanol or isopropanol and diluted to 50 weight percent solids content in the solvent. Two lots each of methyl ester VCAP-75 and methyl ester PMR II50 and one lot each of isopropyl ester VCAP-75 and isopropyl ester PMR II-50 were prepared. PMR II-50 was mixed in a molar ratio of 9 HFDE, 10 PPDA, and 2 NE 
(formulated at $\mathrm{n}$ value of 9, thus providing a numeric FMW of 5047, hence called PMR II-50). The VCAP-75 was mixed in a molar ratio of 15 HFDE, 14 PPDA and 2 PAS (formulated at $\mathrm{n}$ value of 14 , thus providing a numeric FMW of 7874, hence called VCAP-75). Samples of the monomer solutions were aged for up to four years at freezer ($15^{\circ} \mathrm{C}$ ) and room temperatures for HPLC analysis.

Prepregs were made from these same 50 weight percent PMR monomer solutions. Unsized T650-35 graphite fabric was hand impregnated (hand painted with a paintbrush) with the amount of 50 weight percent PMR monomer solutions calculated to generate a 37 weight percent resin laminate after curing. The methanol wet prepreg was allowed to dry overnight to form a pliable prepreg while the isopropanol wet prepreg was allowed to dry up to five days to only still give a wet and tacky prepreg. Both prepregs were then cut into $10.16 \times 10.16$ centimeter $(\mathrm{cm})(4 " \mathrm{x} 4 ")$ plies with some prepregs aged for up to four years at room temperature and some at freezer temperature.

\subsection{HPLC Samples and Analysis}

The initial (unaged) and aged 50 weight percent solutions were diluted to a $0.5 \%$ weight to volume $(\mathrm{wt} / \mathrm{v})$ solution in acetonitrile $(\mathrm{AcN})$ for HPLC analysis. The resin was also analyzed after being leached from the aged prepreg by allowing it to sit in methanol for one hour (a simple study determined one hour was sufficient to dissolve the resin) at a 1:100 wt/v dilution. This was further diluted 1:10 with AcN for HPLC analysis.

HPLC analysis was performed using a Beckman 167 System Gold LC pump connected to a Beckman 167 System Gold variable ultraviolet (UV) detector interfaced with a standard IBM compatible computer. The data processing software is the Maxima package from Waters. HPLC grade AcN from Fisher and Milli-Q filtered distilled water with $0.005 \mathrm{M}$ tetrabutyl ammonium perchlorate (PIC reagent, Fisher) added were used in the mobile phase. A gradient solvent flow of $25 \%$ to $100 \% \mathrm{AcN}$ at $1.0 \mathrm{ml} / \mathrm{min}$ over 40 minutes, a reverse-phase technique, on a Hamilton PRP-1, $250 \mathrm{~mm} \times 4.1 \mathrm{~mm}$, column packed with polystyrene divinyl benzene of $10 \mu \mathrm{m}$ particle size and $75 \AA$ pore size was used.

\subsection{Prepreg Volatile Contents and Prepreg Rheology}

Prepreg volatile contents for the methyl ester composites were determined in triplicate after storage aging but before composite processing using $2.54 \times 2.54 \mathrm{~cm}$ (1"x1") prepreg pieces, and reported as the weight percent of volatile material lost after heating to $204^{\circ} \mathrm{C}$ for one hour (the temperature/time by which most imidization occurs). Prepreg volatile contents for the isopropyl ester composites were determined as single data points from the actual weight of volatiles lost at $371^{\circ} \mathrm{C}$ during composite processing, and reported as the weight percent of volatiles lost during the composite processing cycle. This does not include any B staging weight loss when staging was done in an oven before composite processing.

A relative viscosity for the methanol and isopropanol based PMR prepregs was obtained for comparison using rheology ${ }^{17}$ after various room temperature storage times. The methanol rheological data was obtained using a Rheometrics RMS-800 Rheometer while the isopropanol rheological data was obtained using a TA 2980 Dynamic Mechanical Analyzer (DMA). Both sample types were prepared in the warp direction as a 4 ply $0^{\circ}$ graphite fabric symmetrical lay-up, B staged in a mold at $121^{\circ} \mathrm{C}$ for 1 hour 
under the weight of the top plate of the mold. The methanol sample size was $1.25 \times 6.35$ $\mathrm{cm}(0.5 " \mathrm{x} 2.5 ")$ while the isopropanol sample size was $1.25 \times 2.5 \mathrm{~cm}(0.5 " \mathrm{x} 1 ")$. Torsional rectangular geometry was used for the methanol samples with a temperature ramp from 80 to $450^{\circ} \mathrm{C}$ at $10^{\circ} \mathrm{C} / \mathrm{min}$ while a single cantilever fixture was used at the same temperature ramp for the isopropanol samples. Both samples used a strain of $0.5 \%$ applied with no auto tension or autostrain options at a frequency of 1 hertz $(6.28 \mathrm{rad} / \mathrm{sec})$. Duplicate runs were made when possible. The analyzing software was Rhios version 4.2.2 for the Rheometrics instrument while the DMA used universal analyzer version $2.5 \mathrm{H}$.

\subsection{Composite Preparation}

Six ply, $10.16 \times 10.16 \mathrm{~cm}, 0^{\circ}$ graphite fabric symmetrical layup composites were processed from each lot of prepreg after various long term room temperature storage times. These composites were processed in a 12 ton press using a simulated autoclave vacuum bagging technique with a final pressure of $3.45 \mathrm{MPa}(500 \mathrm{psi})$ and a final temperature of $371^{\circ} \mathrm{C}$. Figure 2 shows the same cycle was used for both the methyl ester PMR systems. Full water aspirator vacuum was applied throughout the entire processing cycle shown. Before processing, the only difference was in using a lower B staging temperature of $149^{\circ} \mathrm{C}$ for the methyl VCAP-75 prepreg versus $204^{\circ} \mathrm{C}$ for the PMR II-50, each for one hour before processing as per Figure 2.

The isopropyl ester processing cycle required more modifications because of the lower isopropanol volatility and higher prepreg volatile content of the isopropanol based prepregs. Figure 3 shows the same final conditions in processing cycles for both isopropyl based systems. Processing differences not seen in the oven staged cycle in Figure 3 result from the staging of the prepregs in an oven at $65^{\circ} \mathrm{C}$ for one hour and then $106,121,135,149$, or $177^{\circ} \mathrm{C}$ for an additional hour. After oven staging, processing then starts with a one hour hold at $204^{\circ} \mathrm{C}$, then a half hour temperature hold at $232^{\circ} \mathrm{C}$ before the final $371^{\circ} \mathrm{C}$ processing. Additional modifications (also not shown in Figure 3) were either staging the isopropyl prepreg in a $65^{\circ} \mathrm{C}$ vacuum oven overnight and/or using a dry ice trap to capture the isopropanol and water evolved by sweeping air through the mold during the heat up before full vacuum was applied when $204^{\circ} \mathrm{C}$ was reached. The latter was used as an attempt to remove the last volatiles rather than entrapping them to create voids when pressure was subsequently applied at $232^{\circ} \mathrm{C}$. In contrast, isopropyl prepregs not staged in an oven initially (also shown in Figure 3), were instead staged in the processing cycle using a series of 3 one hour temperature holds at 65,121 and $177^{\circ} \mathrm{C}$, with pressure and vacuum applied at $260^{\circ} \mathrm{C}$, thereafter both cycles in Figure 3 increase the temperature up to $371^{\circ} \mathrm{C}$ to complete the processing. Thus, the staging during processing cycle always consumed more processing time, but without better results as seen later.

\subsection{Composite Evaluation}

After processing, the quality of the composites was evaluated by ultrasonic Cscan (through transmission, $5 \mathrm{MHz}$ ) to qualitatively determine the amount of voids present. The laminates were then postcured in a forced air oven using a ramp rate of $17.4^{\circ} \mathrm{C}$ per hour for 8 hours starting at $232^{\circ} \mathrm{C}$ to reach the final postcure temperature of $371^{\circ} \mathrm{C}$, where they remained at $371^{\circ} \mathrm{C}$ for 16 hours. Mechanical test specimens 
approximately 6.9 x $0.5 \mathrm{~cm}\left(2.7 " \mathrm{x} 0.2^{\prime \prime}\right)$ were then cut from each methyl ester laminate and from one half of each isopropyl ester laminate. Three point flex tests were done in triplicate according to ASTM D-790 at both room temperature and $316^{\circ} \mathrm{C}$ for each laminate made from each lot of aged prepreg. The only exception to this is the methyl ester specimens made from the six month room temperature stored prepreg, which were inadvertently tested at $288^{\circ} \mathrm{C}$ rather than $316^{\circ} \mathrm{C}$.

The methyl and isopropyl ester laminate end pieces were then cut into three nominally $2 \times 2 \mathrm{~cm}\left(0.8^{\prime \prime} \times 0.8^{\prime \prime}\right)$ pieces. These, along with the cut methyl ester test specimens and the other half of each isopropyl laminate, were aged isothermally at $316^{\circ} \mathrm{C}$ for 1000 hours to determine the percent weight loss. After aging, the larger aged isopropyl pieces were cut into smaller mechanical test specimens. These were used along with the previously cut, $316^{\circ} \mathrm{C}$ aged methyl ester specimens for the same room temperature and $316^{\circ} \mathrm{C}$ flexural tests after the 1000 hours of aging at $316^{\circ} \mathrm{C}$. The weight loss after 1000 hours of isothermal aging at $316^{\circ} \mathrm{C}$ and any change in flexural strength (FS) and modulus (FM) at room temperature and $316^{\circ} \mathrm{C}$ at each storage interval were determined (inadvertently, for the six month methyl ester storage interval, the tests were performed at $\left.288^{\circ} \mathrm{C}\right)$.

Thermogravimetric analysis $\left(\mathrm{TGA}\right.$, ramp rate $\left.=10^{\circ} \mathrm{C} / \mathrm{min}\right)$ and thermomechanical analysis (TMA, $5 \mathrm{~g}$ expansion probe, ramp rate $=10^{\circ} \mathrm{C} / \mathrm{min}$ ) were performed on the nonpostcured and postcured laminates using the smaller end pieces and some of the mechanical tested specimens. Also, acid digestions of the tested specimens and end pieces were performed for quantitative void content determination according to ASTM method D-3171 in addition to the qualitative void content determination by ultrasonic Cscanning of the uncut laminate, done both before and after postcuring.

\section{Results and Discussion}

\subsection{Methyl Ester versus Isopropyl Ester PMR Technology}

\subsubsection{HPLC Comparisons}

Evaluation of the HPLC data from the methyl ester approach (shown in Figure 4) indicates a gradual decrease in the amounts of pure monomers present as the solutions age. The prepregs age slower so they are not shown in Figure 4. There is also a decrease in the rate of disappearance of pure monomers with increasing storage time. The largest drop in this rate occurs within the first two weeks, somewhat leveling-off to a steady rate afterward. This is consistent with the concentration changes and subsequent kinetic effects. ${ }^{18}$ The relative rates of aging product formation can be derived directly from changes in HPLC peak areas. The growth of aging products is consistent with the decrease in pure monomers present. Table I lists the retention times and the appearance times of detectable amounts of aging products found using HPLC.

The room temperature isopropyl ester approach HPLC data indicates a similar trend compared to the methyl ester approach, however at a significantly reduced rate. HPLC data for the methyl ester and isopropyl ester solutions and prepregs aged at freezer temperature $\left(-15^{\circ} \mathrm{C}\right)$ did not show any significant formation of aging products so both the room temperature stored isopropyl ester and all the freezer stored sample HPLC data are not illustrated. The HPLC data clearly indicates the isopropyl ester approach significantly reduces the aging reactions for the monomer solutions and prepregs such 
that it generally takes at least ten times longer to produce the same amounts of aging products as seen in the methyl ester aging. Because the aging products tend to be less soluble, they precipitate during room temperature aging to act as a visible indicator of aging with the methyl esters giving precipitates much faster than the isopropyl esters, as listed in Table II.

Some scatter in both the monomer solution and prepreg samples was seen in the HPLC data. This is due in part to week-to-week instrument variability, but also due to concentration changes associated with solvent (methanol or isopropanol) evaporation from the prepregs and variations inherent for a hand layup prepreg preparation. Further complications arise because of the precipitation of some of the aging products formed when preparing the methanol solution HPLC sample into AcN. The methyl ester aging products increase in concentration and molecular weight, which decreases their solubility in methanol and/or AcN. This became a factor after about 20 weeks for only the room temperature stored methanol based solutions (their prepregs aged slower, thereby avoiding insolubles at that time, so they are not shown in Figure 4). Consequently, all HPLC evaluations were discontinued after twenty weeks as seen in Figure 4. However precipitation was not true for any of the isopropanol solutions and prepregs as they remain fully soluble because they do not react during room temperature aging. Thus, not showing aging, isopropanol HPLC results were also not illustrated in Figure 4.

In spite of these complications, it can be concluded that VCAP-75 resin aging products form as follows: PAS disappears very fast, presumably forming a polystyrene type polymer; next HFDE reacts more slowly with PPDA forming the 1:1 adduct and later the 2:1 and 1:2 adducts. Similarly, the PMR II-50 resin aging products form as follows: NE reacts with PPDA extremely fast to form mononadimide (which is found as a precipitate within a week ${ }^{5}$, see Table II) and later some bisnadimide; next HFDE reacts more slowly with PPDA as in VCAP-75. The pathway to these aging products in methanol using methyl esters is shown in Figure 5. The aging pathways using isopropyl esters and isopropanol instead of methyl ester groups and methanol are identical to Figure 5 so they are not illustrated, however they proceed significantly slower.

\subsubsection{Prepreg Viscosity Comparisons}

Methyl ester prepreg viscosity increases with the formation of aging products. The rheological analysis shows that there is a general shift toward a higher temperature minimum viscosity with increased aging time for the methanol based PMR II-50 prepreg, but this is not quite as clearly seen for the methanol based VCAP-75 prepreg (both compared in Figure 6). Also, both polyimides exhibit a broadening and flattening of melt viscosity with time in the $225^{\circ} \mathrm{C}$ range, indicating that processability is being slowly lost with increasing room temperature storage time. The measured viscosities shown in Figure 6 are relative and not the true viscosity of the resins. They represent a combination of the graphite fabric and the resin. This data is useful though and provides an indication of the maximum and minimum viscosity ranges and any temperature shifts occurring within these viscosity ranges. It is also good as a comparative tool between samples run under identical conditions. Melt viscosity is important because any changes in processability can impact the finished laminate quality.

Different changes with increasing room temperature prepreg storage time for the isopropyl ester based PMR II-50 and VCAP-75 are seen compared to the methyl ester 
systems. Figure 7 shows that the minimum viscosity occurs at a significantly lower temperature (about $170^{\circ} \mathrm{C}$ instead of in the $225^{\circ} \mathrm{C}$ range for methyl ester) and is much broader in the unaged isopropyl ester prepreg compared to the 4 to 12 month aged isopropyl ester prepreg. This correlates well with the much higher processing flow seen in the unaged isopropyl ester prepreg (discussed later in the processing flow comparison section). In fact all of the isopropyl ester prepreg processing flows exceeded that of the methyl esters. Additional data from isopropyl VCAP-75 prepreg that had been aged for 111 months at room temperature shows almost a total loss of the $170^{\circ} \mathrm{C}$ processing viscosity peak. This indicates that its storage life has been grossly exceeded. It also fits in with the later discussion that isopropyl ester prepreg laminates aged for 50 months were inferior to isopropyl laminates aged for twelve months or less.

\subsubsection{Prepreg Volatile Content Comparison}

The initial volatile content of the methyl ester PMR prepreg allowed to dry overnight after the resin solution is painted onto the fiber is typically around 10 to $12 \%$ (Figure 8). During room temperature storage aging there is an initial rapid drop over one month that levels off at about $7 \%$ volatiles for both of the methyl ester based prepregs. This amount of $7 \%$ volatiles represents the remaining volatiles tied up as methyl esters and water to be lost later during conversion to imides during the condensation polymerization (see Figure 5) plus any remaining methanol solvent in the prepregs. The theoretical total volatile content of freshly painted wet prepreg (calculated to contain $37 \%$ resin in the finished laminate) is $21.57 \%$ for methyl ester PMR II-50 and $21.46 \%$ for methyl ester VCAP-75, while the theoretical imidization volatiles in these prepregs are only $6.24 \%$ and $5.93 \%$, respectively. The difference represents the "free" methanol used as a solvent to make the $50 \%$ by weight monomer solutions applied to the fiber. These $<7 \%$ volatiles are not lost until imidization occurs during either the $204^{\circ} \mathrm{C}$ prepreg volatile content determinations, very long term room temperature prepreg aging times ( $>6$ months) or composite processing.

In contrast to the methyl ester systems having a $21.46-21.57 \%$ theoretical volatile content range which dries to the $10-12 \%$ range overnight (see prior paragraph), the analogous isopropyl ester systems initial theoretical volatile content are $22.7-22.9 \%$ but only dry to between $18-19 \%$ (Figure 8 ) after four days of room temperature storage (hereafter called zero months storage time). Again, the difference is from the loss of some of the "free" methanol or isopropanol used as solvents for the initial $50 \%$ monomer solution to paint the prepreg fiber. After 4 months of room temperature storage there is only a slow decrease from the 18-19\% isopropyl volatiles range to the $14-15 \%$ range where it levels off, remaining so for even 50 months of storage time (but not shown past 12 months in Figure 8). This is still much higher than the theoretical 8.74\% and 8.49\% volatile contents loss expected from just imidization into condensation polymerization products of isopropyl PMR II-50 and isopropyl VCAP-75, respectively. This indicates that, unlike the methyl prepregs, the isopropyl prepregs never totally dry out during room temperature storage. Surprisingly, the isopropyl processing volatiles remain as much as $6 \%$ higher than the theoretical imidization volatiles $(<9 \%)$ even after four years of room temperature storage. All the "free" volatiles should have disappeared more rapidly than the volatiles lost from imide formation chemistry as the prepregs dry out further during extended storage. However, the isopropanpl volatiles did not dry out as the "free" 
isopropanol loss during storage is significantly slower than methanol loss, as seen in Figure 8.

Oven staging the unaged isopropyl prepregs was required before processing (see Figure 3) to alleviate the excessive initial isopropyl prepreg volatile contents. Alternatively, the isopropyl prepregs slowly partially dry out during long term aging and become more processable. These then can be processed without oven staging using an increasing series of temperature holds as shown in Figure 3. Oven staging was done by heating the isopropyl prepregs in a forced air oven to $63^{\circ} \mathrm{C}$ for one hour followed by another hour at $121^{\circ} \mathrm{C}$. This usually reduced the volatiles generated during processing to $11-12 \%$ (with one as low as 9\%), starting from a 19.5-21.3\% range without prior storage aging or starting from 13.9 to $15.0 \%$ with prior 50 to 8 month room temperature storage aging, respectively. The volatiles after staging were still at or above the theoretical imidization volatiles (8.49-8.74\%) and always below the minimum 14-15\% range shown in Figure 8 for the unstaged isopropyl prepregs. Surprisingly the $121^{\circ} \mathrm{C}$ staging of aged prepregs only reduced the processing volatiles down to the same $11-12 \%$ level as that obtained for unaged /staged prepregs, i.e. this represents the lower volatile limit after $121^{\circ} \mathrm{C}$ staging irrespective of any amount of prior storage aging time. This lack of change in the volatile content correlated to resin flow will be discussed in the subsequent section.

Additional processing data showed the processing volatile levels after oven staging at $135^{\circ} \mathrm{C}$ were still $14.88-14.84 \%$ (slightly higher than the $11-12 \%$ obtained after $121^{\circ} \mathrm{C}$ staging, but done with a new prepreg batch in a different oven at a different time so the overall data trend still does agree). Attempts to lower to initially high volatile contents by instead using a $65^{\circ} \mathrm{C}$ vacuum oven overnight still needed additional $121^{\circ} \mathrm{C}$ oven staging to provide similar volatile contents so only oven staging at various temperatures was investigated further. Additional processing data showed more intensive staging at even higher temperatures (in place of the $121^{\circ} \mathrm{C} /$ one hour hold) was needed to further dry the prepregs, with volatiles now going below the $9 \%$ imidization theoretical volatile level. At higher staging temperatures, more imidization volatiles were finally removed as shown by the much lower volatiles remaining were only $2.63-3.60 \%$ at $149^{\circ} \mathrm{C}$ and $2.10-2.63 \%$ at $177^{\circ} \mathrm{C}$ for isopropyl PMR II-50 and isopropyl VCAP-75, respectively. A benefit of oven staging at any temperature tried from 106 to $177^{\circ} \mathrm{C}$ was that the unaged prepregs were considerably less tacky to even nontacky after the higher staging temperatures.

\subsubsection{Composite Processing Resin Flow Comparison}

Not surprisingly, the results from HPLC evaluations of the prepregs and solutions indicate that room temperature solutions age much faster than the prepregs. This is most likely due to the greater mobility of the monomers in the solution versus the prepreg. The freezer stored $\left(-15^{\circ} \mathrm{C}\right)$ methyl solutions and methyl prepreg materials did not age over the time studied indicating that freezing is an excellent storage method. However, at room temperature an increase in the presence of aging products seen in the HPLC corresponds to noticeable changes in the viscosity of the prepregs (see rheology, Figure 6). This aging of methyl prepregs would be expected to necessitate use of higher processing temperatures and a narrower processing window as was observed (see Figure 2 versus Figure 3). Thus, unique processing conditions would be required for each aged (stored) solution or prepreg, which is not a desirable quality. The insignificant aging of 
isopropyl ester systems, however, compared to the methyl ester systems, eliminates the need for freezer storage.

The lower rate of isopropyl storage aging is in agreement with the rheology (Figure 7) and HPLC data discussed earlier. This is also in agreement with the processing resin flow data shown in Figure 9. This Figure shows the amount of resin flow as a percent of laminate weight squeezed into the bleeder plies for the methyl and isopropyl based resins, determined from the weight gains in the bleeder cloths. The methyl ester composites were processed as per Figure 2 and the isopropyl as per Figure 3, both without prior oven staging. The eight month isopropyl ester composites were processed as per Figure 3 with prior oven staging, thus causing the lower flow as shown by the minimum in the isopropyl resin bleeds at the 8 months point in Figure 9.

The most important thing to note in Figure 9 is methyl VCAP-75 loses almost all its processing flow in the first month of prepreg storage time while methyl PMR II-50 takes 4 months to lose a comparable amount of flow. It cannot be attributed to solvent drying out since both do so at the same rate (see Figure 8). This difference is probably due to the PAS endcap found in VCAP-75. PAS is very reactive and disappears within the first day of room temperature storage as seen by HPLC. This endcap reactivity, in combination with subsequent imidization during the first month of methyl ester VCAP75 storage, would cause the molecular weight to rapidly increase and processing flow to decrease more rapidly compared to the methyl ester PMR II-50.

This same drop in processing flow should be observed for isopropyl VCAP-75, however, the imidization aging reaction is considerably slowed down by the use of isopropyl esters so the resin flow curves of isopropyl VCAP-75 and isopropyl PMR II-50 are virtually identical for almost the entire long term extended storage time (Figure 9). These isopropyl flow curves look very similar to the processing flow curve of methyl ester PMR II-50 for the first two months of storage. The methyl ester PMR II-50 ages faster by solvent loss (Figure 8) and imidization (HPLC, Figure 4) causing the flow curves to finally diverge from the isopropyl curve after two months of storage time (Figure 9), thereafter dropping below the almost nonexistent methyl PMR II-50 flow range at the four months aging time, also shown in Figure 9. In contrast, the isopropyl prepregs can only lose flow during the first two months of storage due to the slower loss of isopropanol and additionally, for isopropyl VCAP-75, by the reaction of the PAS endcap. The initial high processing flow of isopropyl prepregs became more manageable after they were aged for four months, a consequence of the reduced volatile content (Figure 8) during aging.

The last interesting point is that while oven staging did lower the volatile contents of unaged and aged isopropyl prepregs, the aging of the isopropyl ester systems did not lower their volatile contents. The aging provided nearly the same volatile contents irrespective of the storage time (see in Figure 10 the upper four lines of unaged versus aged volatile contents being only slightly higher and the two lower lines of unagedstaged versus aged-staged (three data points each) being at the same volatile content level). However, the processing flow after mild oven staging did not significantly decrease from the flow of the respective unaged or aged processing flows without staging (compare the four upper lines above their respective aged and staged lower lines being at the same resin flow amounts in Figure 10). The flow was not significantly reduced at mild staging temperatures (up to $121^{\circ} \mathrm{C}$ ) for unaged isopropyl prepreg, being in the 8.63- 
$15.11 \%$ range for staged prepregs compared to $6.85-17.58 \%$ for unstaged prepregs, nor was flow reduced for aged prepregs as their flow was already minimal, with flow being $0.65-1.92 \%$ for staged prepreg compared to $0.41-6.88 \%$ for unstaged prepregs. At even higher staging temperatures (not shown in Figure 10 but staged at $149^{\circ} \mathrm{C}$ and $177^{\circ} \mathrm{C}$ ) the flow was reduced to $0 \%$ regardless of the age of the isopropyl prepregs. Figure 9 showed isopropyl prepregs lose most of their processing flow by four to eight months of aging via the normal aging process so added oven staging is not needed. The correlation plot in Figure 10 of isopropyl prepreg volatiles versus isopropyl resin flow clearly shows the utility of all this. It indicates the isopropyl prepregs are less susceptible to accidental temperature excursions than the methyl prepregs because they retain processability, with or without oven staging at up to $121^{\circ} \mathrm{C}$ and irrespective of whether or not they had additional room temperature aging before the temperature excursion. This fact could be useful in hot melt manufacturing processes and in preventing aging accidental damage during manufacturing, shipping, handling, transportation and storage.

\subsubsection{Composite Quality Comparison}

Evaluation of the processed methyl laminates using ultrasonic C-scan results show that all the panels were of acceptable quality with a slight decrease in C-scan quality with increasing prepreg storage time at room temperature (Figures 11-12). The lighter colors indicate lower void volumes than the darker colors indicate. Both lots of each methyl resin went from white-red to red-yellow over 6 months prepreg storage time, indicating slightly higher void contents with increased prepreg storage time. Acid digestion results (Table III) showed a low level of voids $(<3 \%, \operatorname{most}<2 \%)$ over the entire six months prepreg storage time. Evidently the chosen methyl ester processing cycle is capable of accommodating some variability in the starting materials when processing flat laminates. However, shaped or curved composite structures would be expected to be less forgiving.

Similar C-scan results were found for both of the isopropyl systems for the laminates prepared with prepregs stored 4 months or more at room temperature (Figure 13). However, the acid digestion results (Table IV) of these composites showed a higher level of voids ( $<4 \%$ for isopropyl VCAP-75 and 6-7\% for isopropyl PMR II-50) than for the analogous methyl ester composites. Without the 4 months or more room temperature aging, the isopropyl VCAP-75 laminates were clearly unacceptable. They showed in Figure 13 a large white no image transmission area except for minimal black spots barely transmitting. Acid digestion results showed 16 to $20 \%$ voids in Table IV while the isopropyl PMR II-50 laminates had satisfactory void contents but excessive resin flow caused higher fiber volumes (Table IV). Surprisingly, the void volumes for isopropyl PMR II-50 did not change significantly over the twelve months aging period (already being scattered in the 4-7\% range during the twelve months) while the void volumes for isopropyl VCAP-75 did change significantly from 16-20\% initially to after 4 months aging to result in a much lower 3-4\% void range. The net effect of excessive initial flow for isopropyl PMR II-50 and high voids for isopropyl VCAP-75 is 4 months of aging was needed for both before satisfactory composites could be fabricated.

Attempts to improve the processing of both unaged isopropyl prepregs by passing air through the simulated autoclave mold during heatup and/or using a dry ice trap on the vacuum line still failed to remove the last imidization volatiles in order to 
obtain lower void contents and/or reduce the excessive initial resin flow. For both isopropyl PMR II-50 and VCAP-75, the mass transport of the isopropanol and water volatiles with these added processing modifications still was not successful. This was surprising because these same processing modifications were successful for fabricating graphite fiber unidirectional and cloth fabric first generation polyimides, i.e. isopropyl PMR-15 and BAX PMR-15. ${ }^{12-14}$ It is believed that the wider processing window of these lower FMW first generation PMR materials allowed their isopropyl esters to be successfully processed into composites. However the same processing modifications were unsuccessful for the higher FMW second generation type PMR isopropyl ester materials because of the narrower second generation processing window. At best the $\mathrm{C}$ scan images only showed the four corners to process into void free areas with the large center areas not even providing an acceptable $\mathrm{C}$-scan image, indicating high voids caused by the unremoved volatiles. In summary, isopropyl ester laminates were of comparable quality to those obtained using state of the art methyl ester PMR technology only after their prepregs were allowed to dry for at least four months at room temperature. All attempts at processing modifications of the unaged isopropyl prepregs still failed to improve upon their quality.

\subsubsection{Composite Thermal Analysis Comparison}

The TMA and TGA data results, given in Table $\mathrm{V}$, did not show a significant change in $T_{g}$ and $T_{d}$ with either increased room temperature storage time or which ester approach used. The average $\mathrm{T}_{\mathrm{g}}$ of the methyl versus isopropyl PMR II-50 ranged from 348 to $362^{\circ} \mathrm{C}$ versus 354 to $368^{\circ} \mathrm{C}$ while the methyl versus isopropyl VCAP-75 ranged from 326 to $344^{\circ} \mathrm{C}$ versus 323 to $384^{\circ} \mathrm{C}$. The average $\mathrm{T}_{\mathrm{d}}$ of the methyl versus isopropyl PMR II-50 ranged from 517 to $540^{\circ} \mathrm{C}$ versus 514 to $540^{\circ} \mathrm{C}$ while the methyl versus isopropyl VCAP-75 ranged from 528 to $538^{\circ} \mathrm{C}$ versus 526 versus $539^{\circ} \mathrm{C}$. These ranges, not considering storage times, but along with their standard deviations in Table $\mathrm{V}$ precluded finding any noticeable trends, thereby any differences are statistically not relevant.

The isothermal aging at $316^{\circ} \mathrm{C}$ for 1000 hours (Figure 14) show that the methyl PMR II-50 laminates made from prepreg stored 0 to 6 months lost between 1.58 and $2.47 \%$ of their initial weight while the isopropyl PMR II-50 laminates made from prepreg stored 4 to 12 months lost between 1.96 and 3.36\% (large specimen size) to $4.96 \%$ (smaller specimen size shown in Figure 14). The unstored isopropyl PMR II-50 prepreg produced inferior laminates that had a higher (5.01\%) weight loss. Beyond twelve months of room temperature prepreg storage, i.e. 50 months storage, the isopropyl PMR II-50 composites showed a much higher $6.89 \%$ weight loss, presumably due to a poor quality laminate. In contrast, the isothermal aging at $316^{\circ} \mathrm{C}$ for 1000 hours (Figure 15) shows that the methyl VCAP-75 laminates made from prepreg stored 0 to 6 months lost between 1.44 and $2.37 \%$ of their initial weight while the isopropyl VCAP-75 laminates made from prepreg stored 0 to 12 months lost between 1.54 and $2.51 \%$. (Note; 2.51 is surprisingly low for zero months considering the poor laminate quality seen via $\mathrm{C}$-scans and acid digestion). Beyond twelve months of room temperature prepreg storage, i.e. 50 months, the isopropyl VCAP-75 composite showed a higher $4.27 \%$ weight loss, presumably due to a poor quality laminate. After four years of prepreg storage the oven 
aging weight loss of both isopropyl laminates had the highest weight loss for all prepreg storage times. This suggests the useful shelf life has finally been exceeded.

A trend of increasing composite thermal oxidative weight loss with increasing prepreg storage time was apparent in the oven aging weight loss data (Figures 14-15) when six months of storage was reached for both methyl prepregs, when twelve months was reached for the isopropyl PMR-II 50, and when beyond twelve months was reached for the isopropyl VCAP-75. At these times both ester systems were now well beyond reasonable normal storage times (baring shipping, handling, transportation and storage accidents). The weight loss data infers that the room temperature prepreg storage limit without subsequent serious increased composite aging damage is about four months for methyl ester based prepregs and over twelve months for isopropyl ester prepregs.

\subsubsection{Composite Mechanical Property Comparison}

Mechanical tests show the expected large decrease in flexural strength (FS) (30 to $50 \%$ ) and flexural modulus (FM) (5 to $20 \%$ ) between room temperature tests and $316^{\circ} \mathrm{C}$ tests and between unaged samples and 1000 hour aged samples. A significant difference in FS and FM was not detected between the laminates made from methyl prepregs stored for zero to four months at room temperature (Figures 16-19) until after six months when the mechanical properties tend to be lower. (Note: 1000 hour oven aged specimens were inadvertently tested at $288^{\circ} \mathrm{C}$ instead of $316^{\circ} \mathrm{C}$, thus artificially raising the already visibly lower mechanical strengths shown). For the corresponding isopropyl systems decreases in FS and FM are barely noticed until after twelve months prepreg storage. This data, like the weight loss data, indicates the useable prepreg storage time/shelf life is about four months at room temperature for methyl esters and more than twelve months for the isopropyl ester PMR systems.

\subsection{Summary of Comparisons}

Comparing the two ester systems data, the HPLC analysis showed that there is a significant amount of aging product formation in room temperature stored methyl prepreg. The rheological data also showed methyl prepreg aging as a shift toward a higher temperature minimum viscosity with increasing storage time (about $15^{\circ} \mathrm{C}$ for PMR II-50 and $10^{\circ} \mathrm{C}$ for VCAP-75). The isopropyl systems do not show these changes in the HPLC or rheological data indicating a lack of aging during storage. It also appears from the composite quality analysis of the methyl PMR II-50 and VCAP-75 laminates that there is a serious decrease in the quality of the finished composites after six months of room temperature prepreg storage. The methyl ester laminate processing windows appear to be broad enough to only accommodate the changes in the prepregs during the first six months of aging.

Instead for the isopropyl ester laminates, which showed a lack of aging in the HPLC and rheological data, the processing window now remains constant for up to 12 months of room temperature storage, once the prepreg is either partially dried by four months of room temperature storage, oven staged before processing or additionally staged within the process cycle. This greater constant processing window happens primarily because significant aging is not seen for the isopropyl prepregs when looking at it as two different isopropyl laminate processing techniques; oven staging used primarily for the shorter prepreg storage times to control volatile content and excessive processing 
flow (as processed in Figure 3) or autoclave staging in discrete steps and temperature holds used primarily for longer prepreg storage times (also in Figure 3).

\section{Conclusions}

The results of this study show that after six months of room temperature storage a determental effect is seen on the composite quality of the methyl ester system for PMR II-50 and VCAP-75 polyimides. However, this time frame extends to at least a year for isopropyl PMR II-50 and isopropyl VCAP-75 polyimides. Freezer storage is still preferable for both ester systems because it creates an added safety margin against product failure. The methyl and isopropyl ester type solutions or prepregs can be stored in the freezer for an indefinite period of time without noticeable formation of aging products. But, if freezer storage is not possible, the results indicate that finite room temperature storage can be an acceptable option.

The major advantage of the isopropyl ester is the protection afforded if overheating during prepreg manufacturing happens, accidental aging in transit occurs, the freezer fails or the resin is used in room temperature stored repair kits. However some processing considerations are required. The major disadvantage of the isopropyl ester is considerable extra effort in processing time at intermediate temperatures is needed to remove the processing volatiles (a mass transport problem) before comparable quality laminates can be obtained. Attempts to do so with B staging in ovens, adding temperature holds during heatup, trapping volatiles with dry ice and/or other volatile removing techniques, e.g. prepreg aging, always made the composite processing longer and more tedious. In conclusion, comparable results with both ester systems can be obtained, but additionally the isopropyl ester system provides a more forgiving processing window in the event of mishandling or improper storage of prepregs because the isopropyl esters age significantly slower.

\section{$\underline{\text { References }}$}

1. Serafini, T.T.; Delvigs, P.; and Lightsey, G.R., Thermally Stable Polyimides for Solutions of Monomeric Reactants, Journal of Applied Polymer Science, 1972, Vol. 16, pp. 905-915.

2. Vannucci, R.D.; Malarik, D.C.; Papadopoulos, D.S.; and Waters, J.F., Autoclavable Addition Polyimides for $371^{\circ} \mathrm{C}$ Composite Application, NASA TM-103223, 1990.

3. Vannucci, R.D., PMR Polyimide Composition for Improved Performance for Improved Performance at $371^{\circ} \mathrm{C}$, NASA TM-88942, 1987.

4. Roberts, G.D.; and Vannucci, R.D., Effect of Solution Concentration and Aging Conditions in PMR-15 Resin, SAMPE Journal, March/April 1986, Vol. 22, No. 2, pp. 24-28, and p. 213.

5. Lauver, R.W.; Alston, W.B.; and Vannucci, R.D., Stability of PMR Polyimide Monomer Solution, NASA TM-79063, 1979. 
6. Scheiman, D.A.; Alston, W.B.; Sutter, J. K.; and Turk, M.J., Characterization of PMR Monomer Solutions and Prepregs Using HPLC Analysis, HITEMP Review 1993: 6th Annual HITEMP, NASA CP-19117, 1993, pp. 18-1 to 18-10.

7. Scheiman, D.; Alston, W.B.; Sutter, J.K.; McCorkle, L.S.; Crane, E.A.; Kelly, A.M.; and Papadopoulos, D.S., VCAP-75 and PMR II-50 Aging Study, HITEMP Review 1994: 7th Annual HITEMP, NASA CP-10146, 1994, pp. 13-1 to 13-11.

8. Crane, E.A.; Scheiman, D.A.; Alston, W.B.; Sutter, J.K.; McCorkle, L.; and Kelly, A.M., VCAP-75 and PMR II-50 Prepreg Stability Study, Proceedings of Annual HITEMP Review 1995: NASA CP-10178, 1995, pp.9-1 to 9-11.

9. Scheiman, D.A.; Alston, W.B.; Sutter, J.K.; McCorkle, L.S.; and Crane, E.A., VCAP and PMR II Solution and Prepreg Aging Study, Proceedings of High Temple XV Workshop, Santa Fe, New Mexico, January 16-19, 1995, pp. D-1 to D-20.

10. Scheiman, D.A.; Crane, E.A.; Alston, W.B.; Sutter, J. K.; and McCorkle, L., VCAP75 and PMR II-50 Prepreg Stability Study, Proceedings of High Temple XVI Workshop, Orange Beach, Alabama, January 29-February 1, 1996, pp. M-1 to M-23.

11. Alston, W.B.; Scheiman, D.A.; and Sivko, G., Second Generation Type PMR Solution and Prepreg Stability Study Using an Alternative Isopropyl Ester Approach, Proceedings of High Temple XX Workshop, San Diego, California, January 24-27, 2000, pp. J-1 to J-11.

12. Alston, W.B.; Gahn, G.S.; and Scheiman, D.A., Solution Stability Study For Two Novel PMR Type Resins, Proceedings of High Temple XVIII Workshop, Hilton Head, South Carolina, January 20-22, 1998, pp. K-1 to K-26.

13. Scheiman, D.A.; Alston, W.B.; Sivko, G.; and Gabriel, C., PMR Solution Solubility and Stability Study for Selected Diamines, HITEMP Review 1999, NASA CP-1999208915-VOL 1, 1999, pp. 6-1 to 6-13.

14. Scheiman, D.; Alston, W.B.; Gahn, G.: and Gabriel, C., Solution Solubility and Stability Study, Proceedings of High Temple XIX Workshop, Denver, Colorado, February 1-4, 1999, pp. T-1 to T-17.

15. Johnston, James C.; Meador, Mary Ann B.; and Alston, William B., A Mechanistic Study of Polyimide Formation from Diester-Diacids, Journal of Polymer Science, Part A: Polymer Chemistry, 1987, Vol. 25, No. 8, pp. 2175-2183.

16. Alston, William B.; and Sivko, Gloria S., Composition and Process For Retarding the Premature Aging of PMR Monomer Solutions and Prepregs, U. S. Patent 6,103,864, August 15, 2000.

17. Kranjc, M.; and Roberts, G.D., Viscosity Measurements of Polyimide Resins, HITEMP Review 1992: 5th Annual HITEMP, NASA CP-10104, 1992, pp. 12-1 to 12-12. 18. Roberts, G.D.; and Lauver, R.W., Quantitative Analysis of PMR-15 Polyimide Resin By HPLC, Journal of Applied Polymer Science. 1987, Vol. 33, pp. 2893-2913.

\section{SUGGESTED KEYWORDS}

Polyimide Composites

High Performance Polymers

Composite Thermal and Mechanical Properties

PMR II-50 \& VCAP-75

Solution and Prepreg Shelf Life 

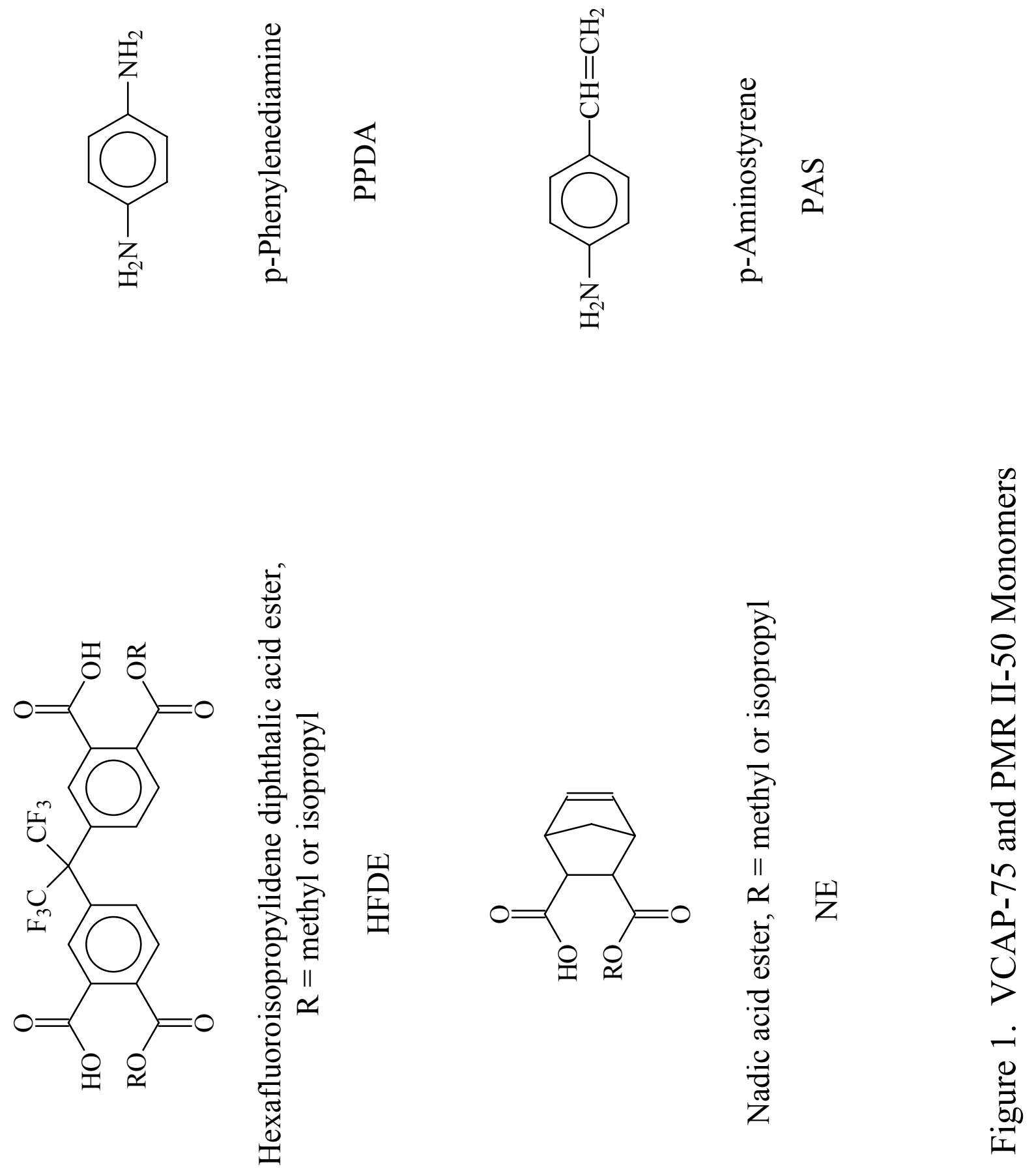


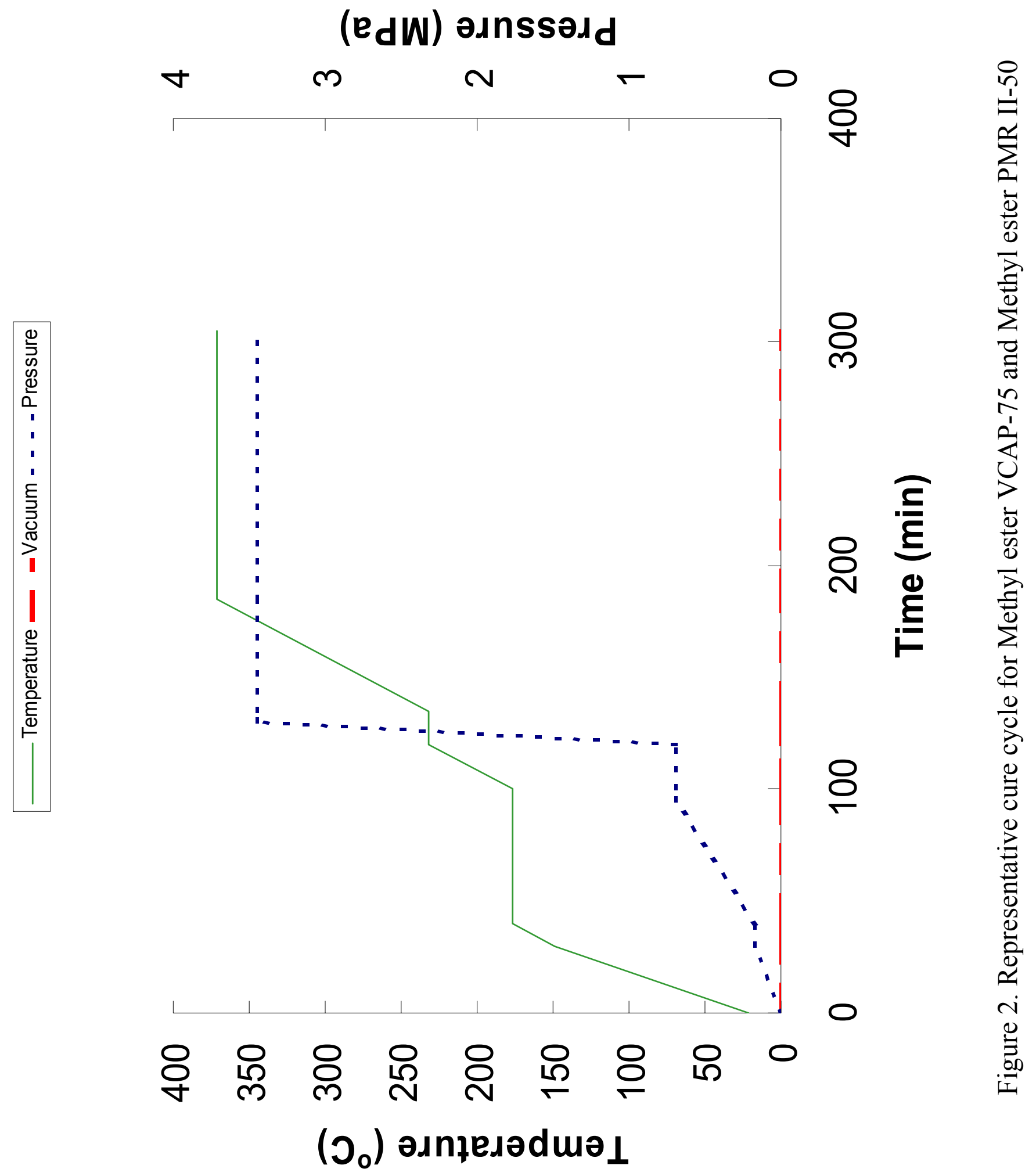




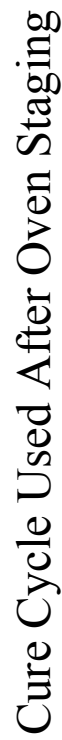

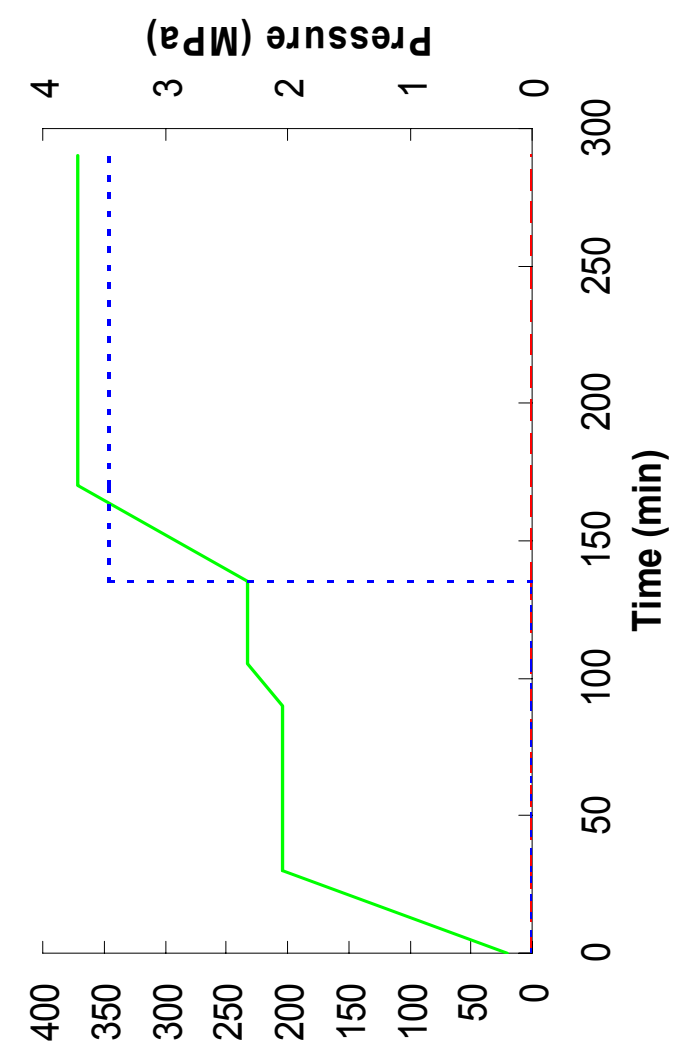

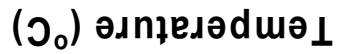

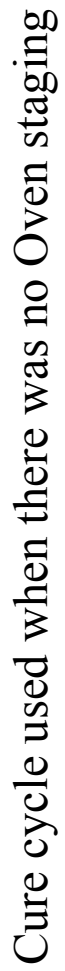
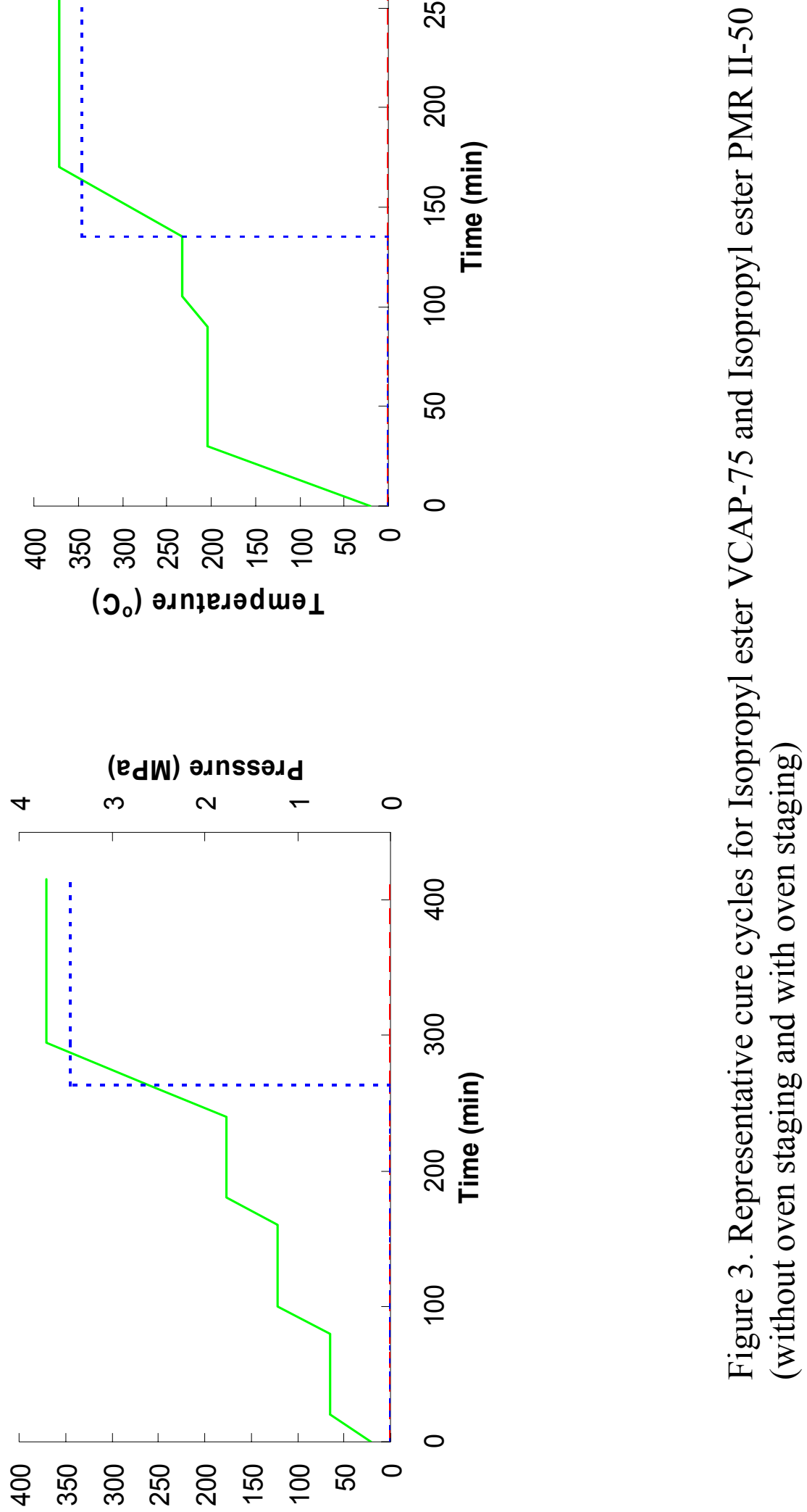

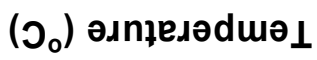



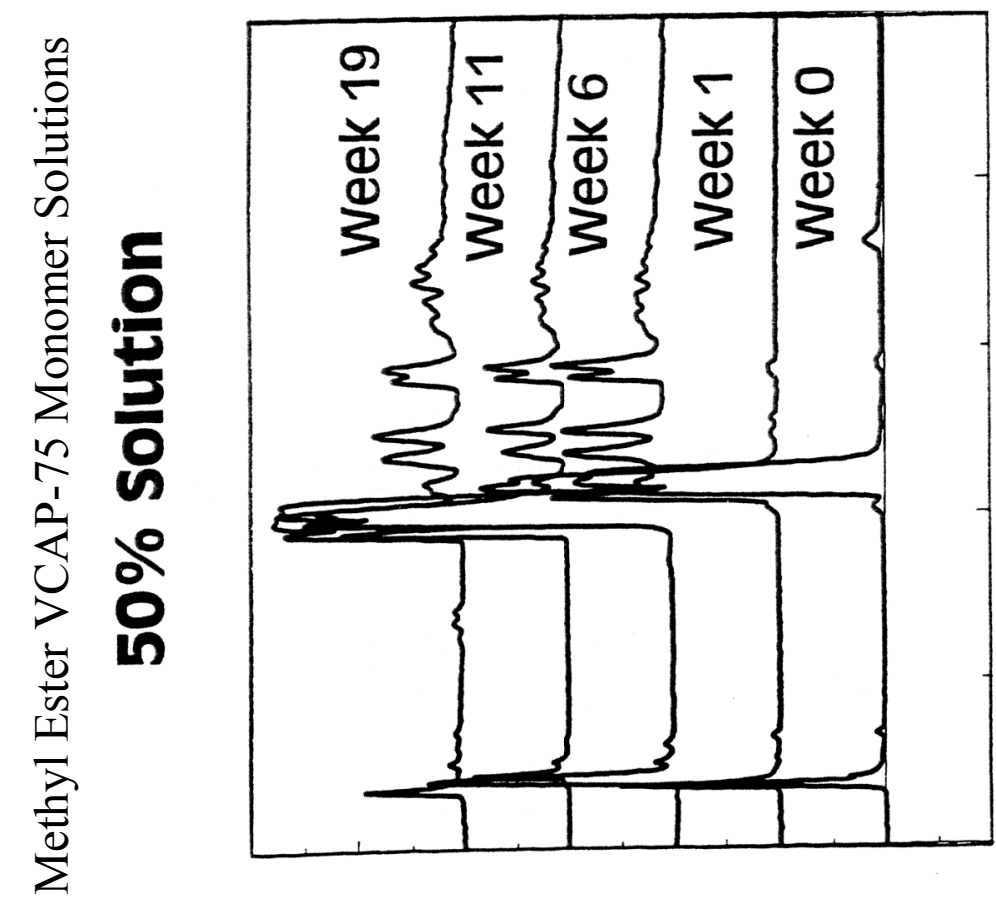

.

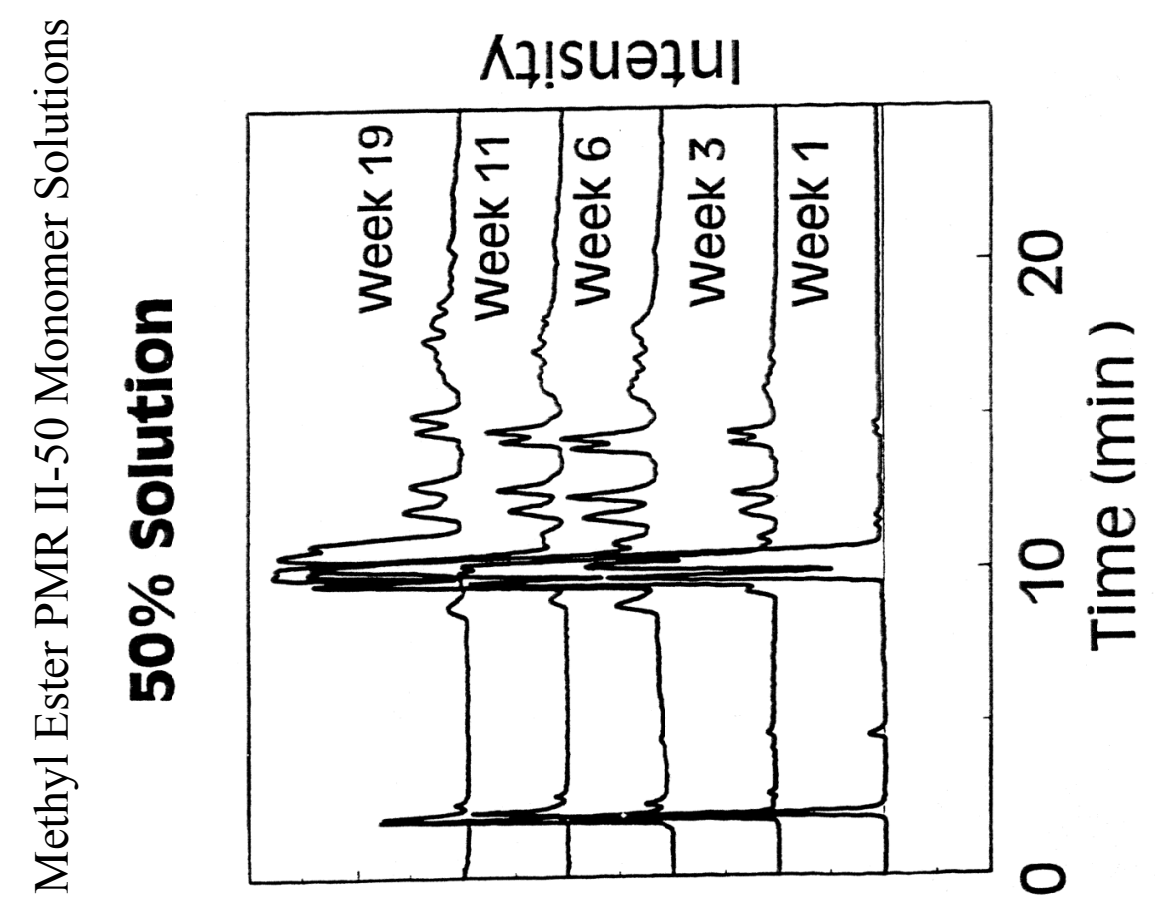

葛

हี

กำ

อั

范

告

$\cup$

的

I

n.

28

Ч

$\nabla$ क

릉

유ㄴㅠㅠ

$\stackrel{\sqcup}{\exists}$

또

$\sum_{0}$

응

극

突

$\sum$

$\forall \cdot \overline{0}$

总 


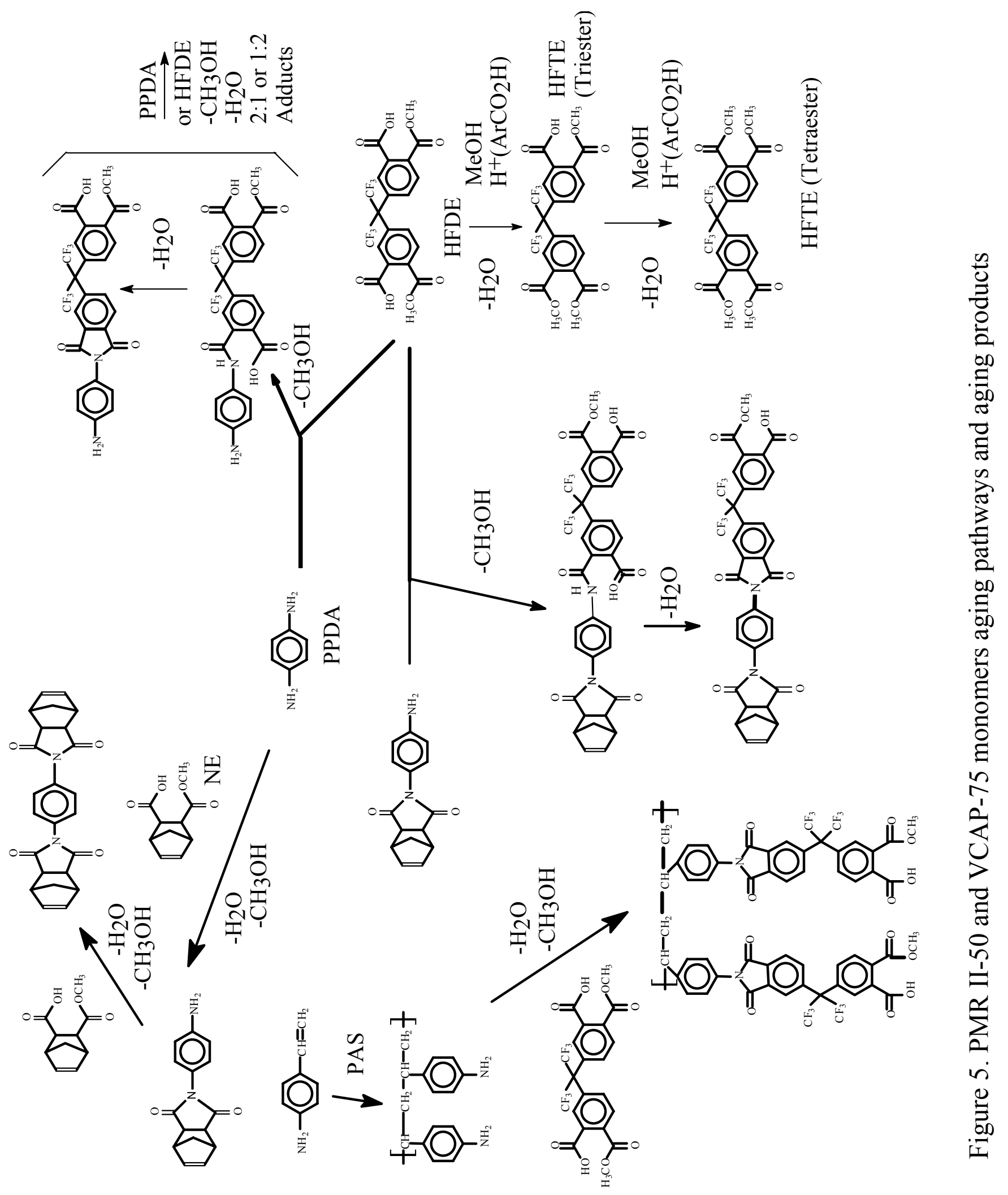




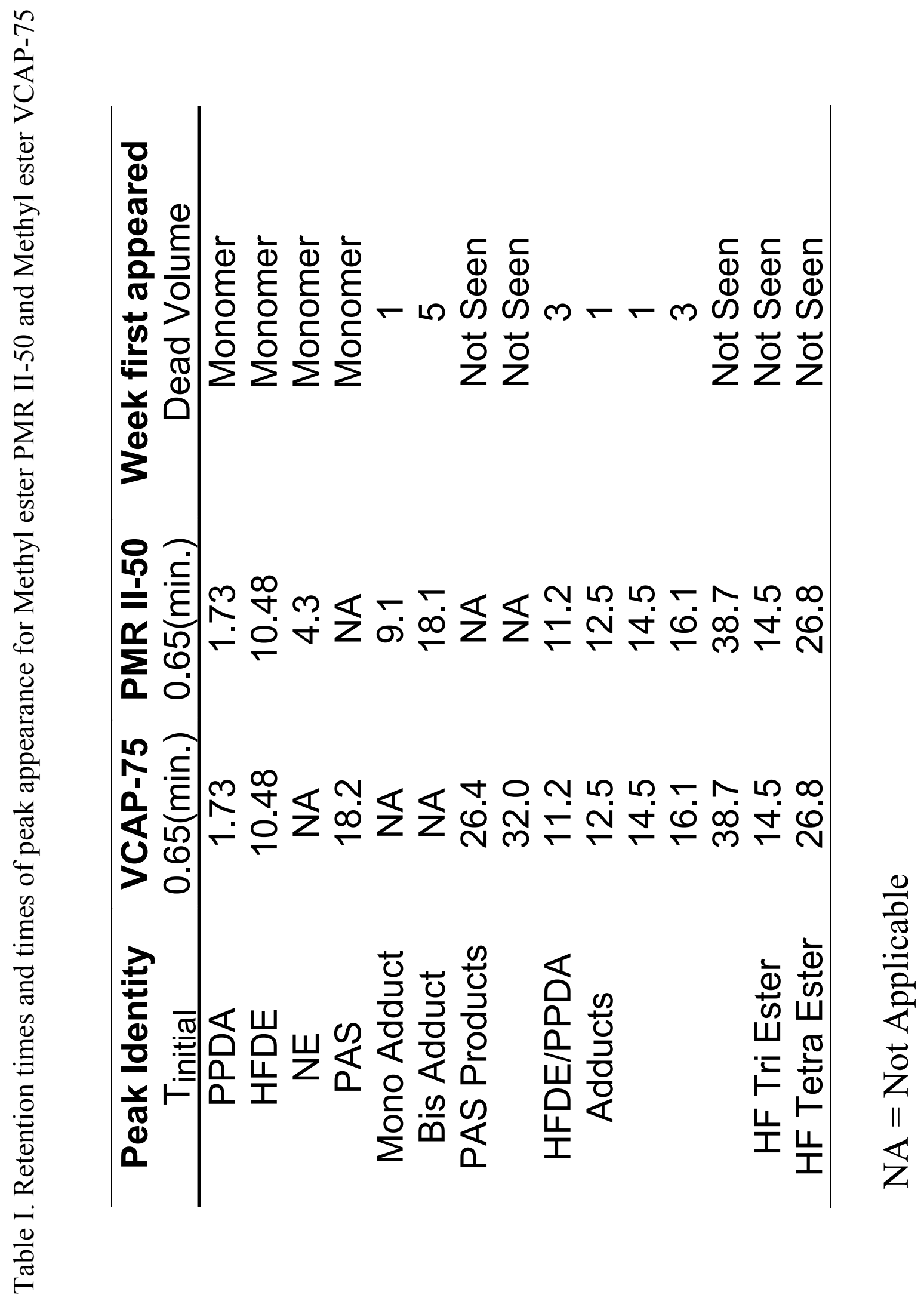



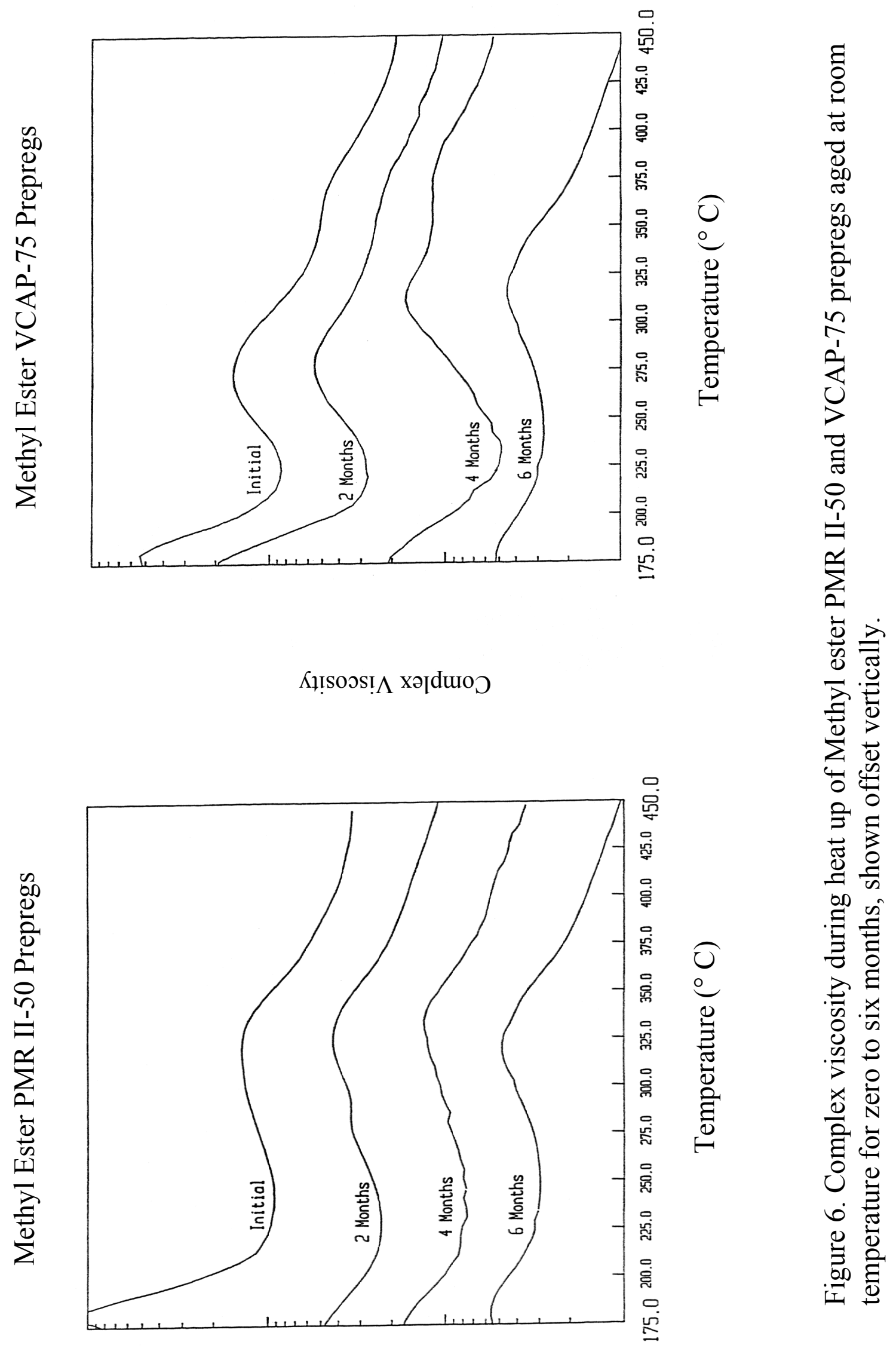

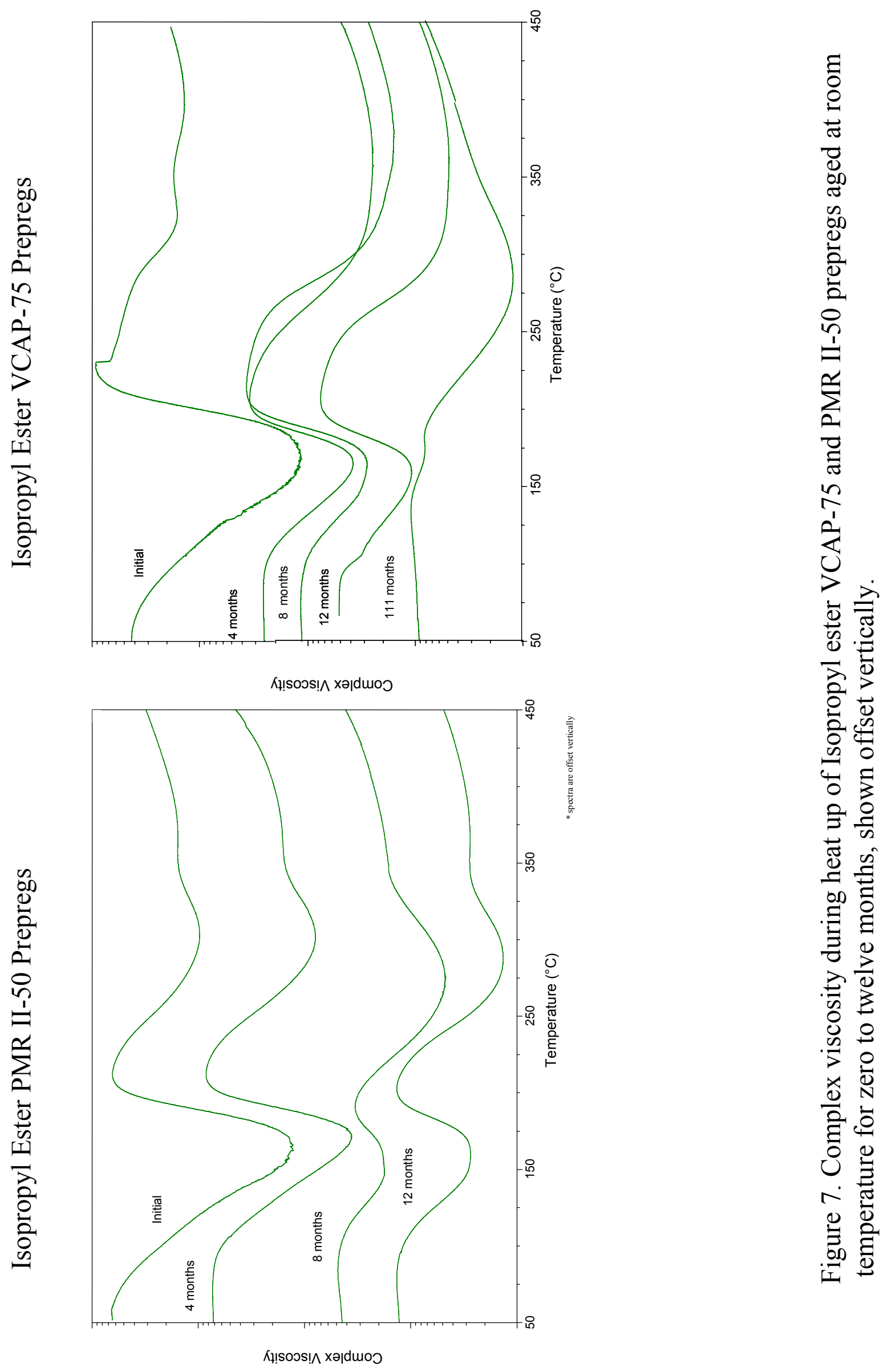

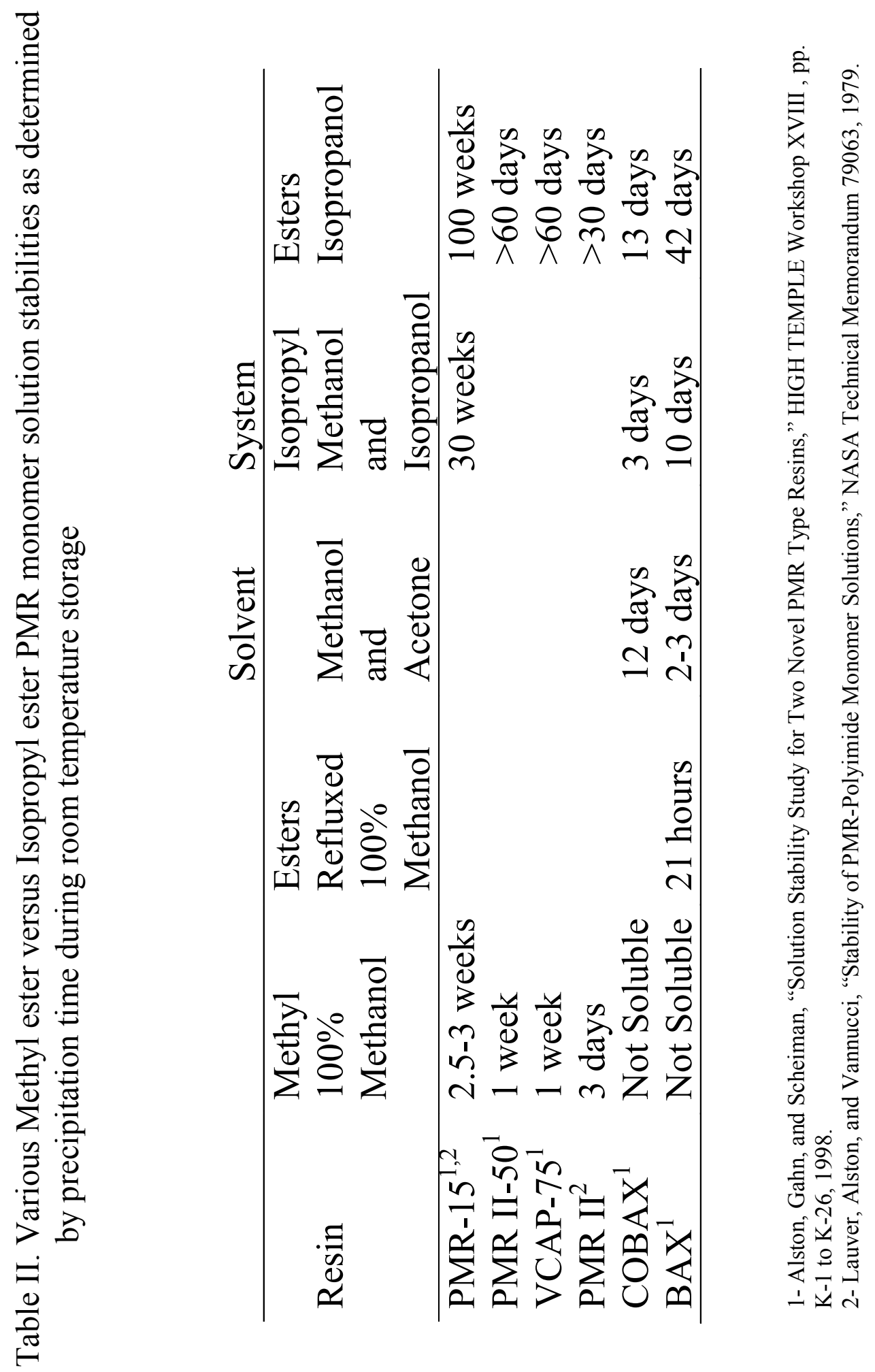

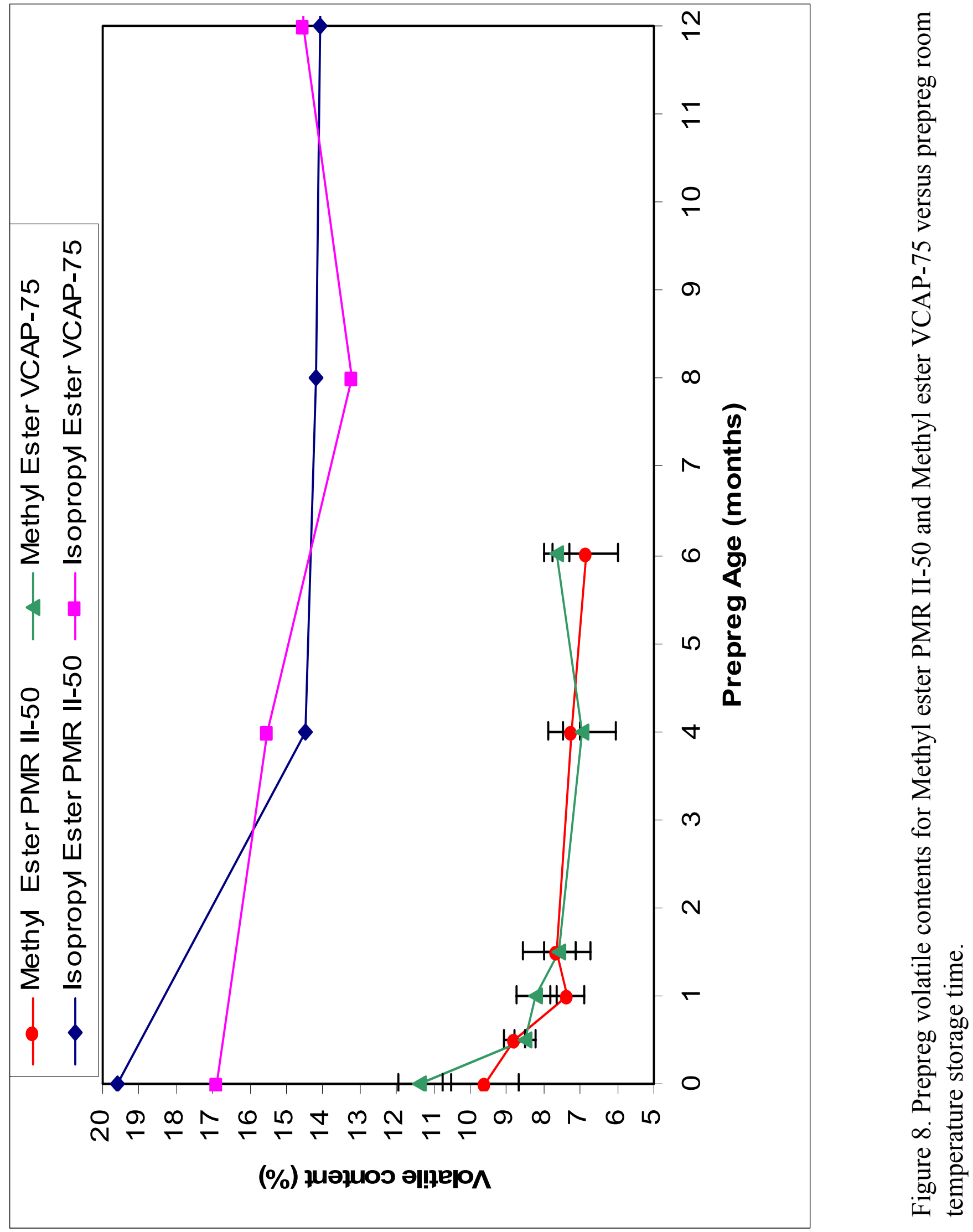


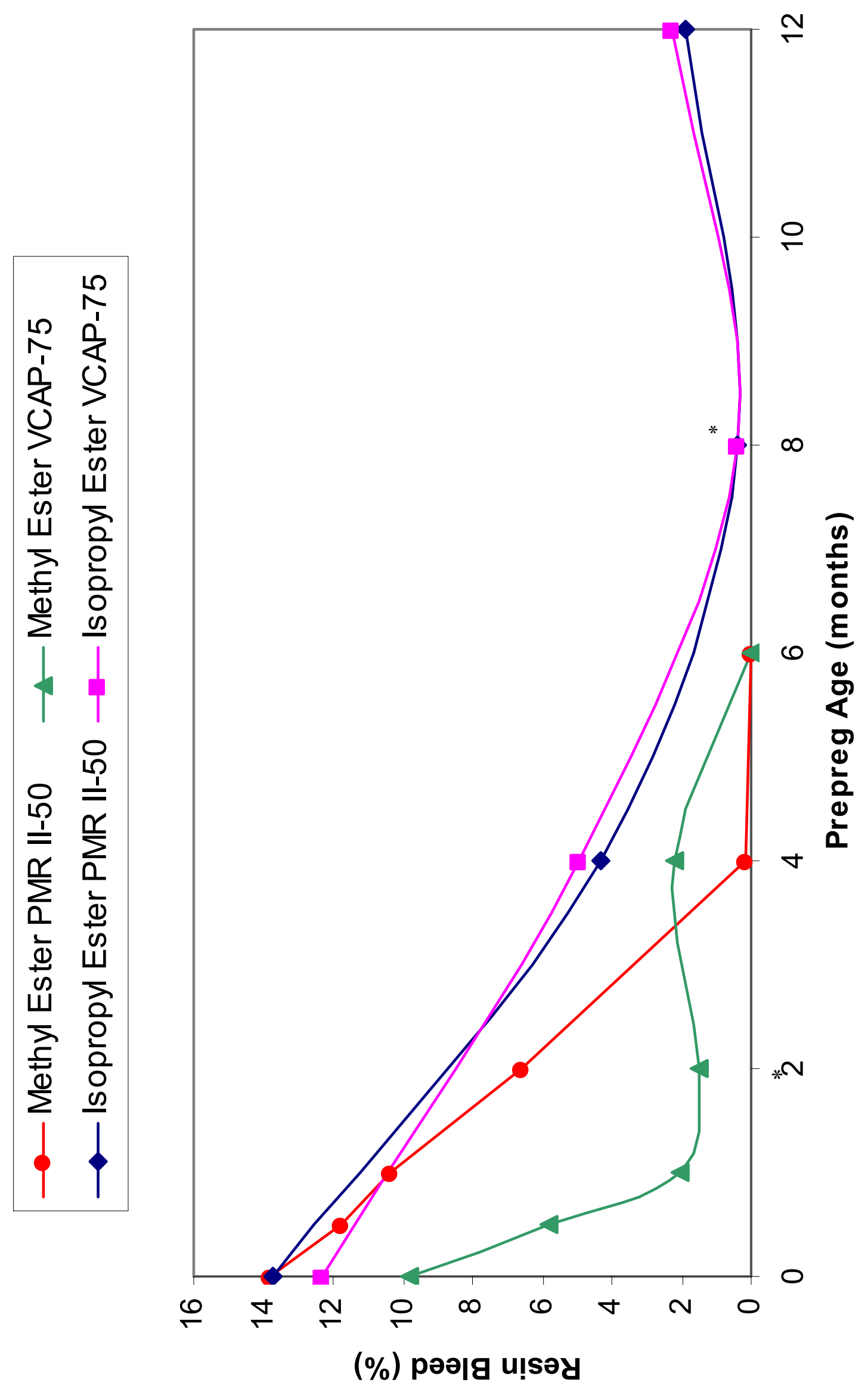




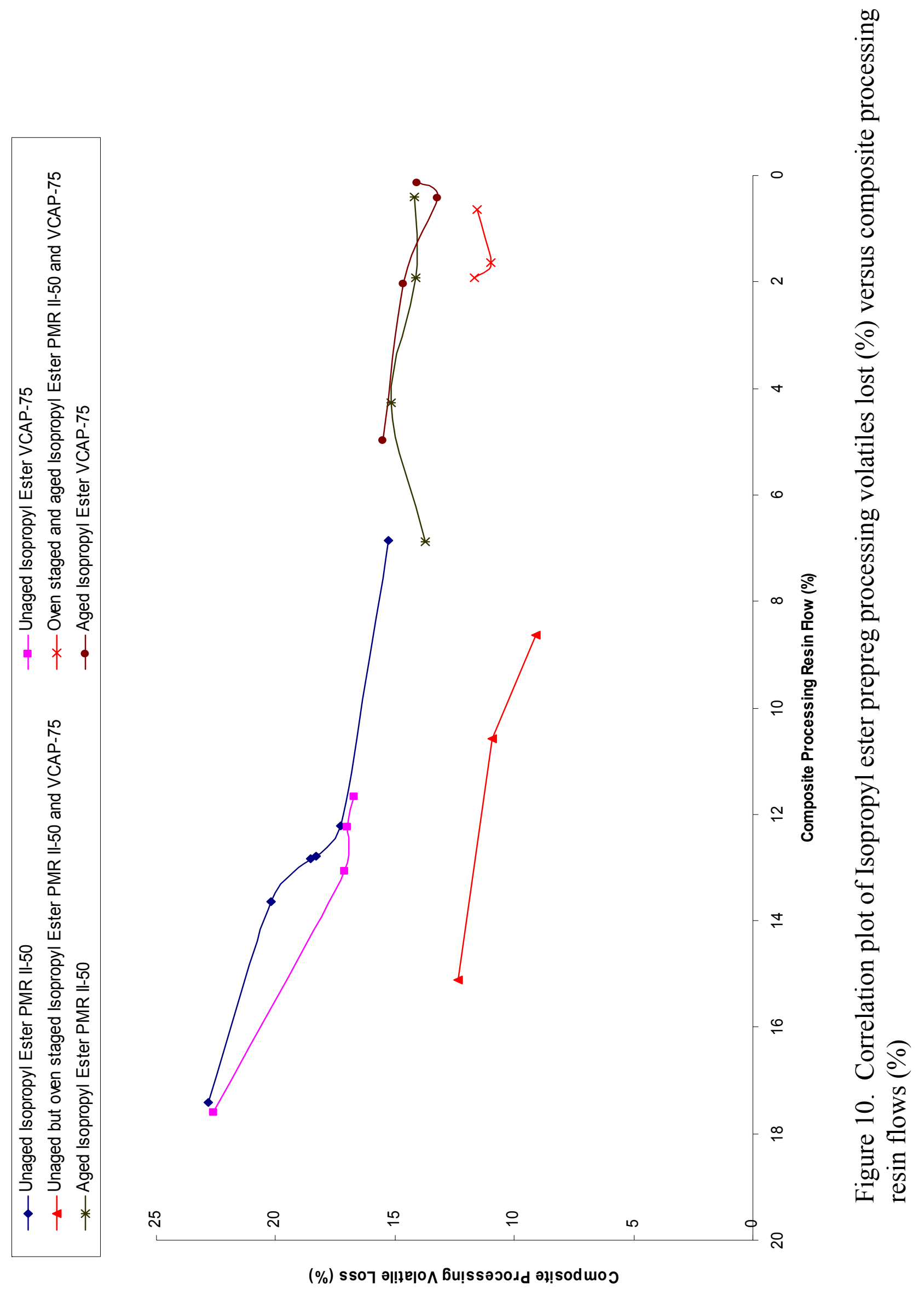




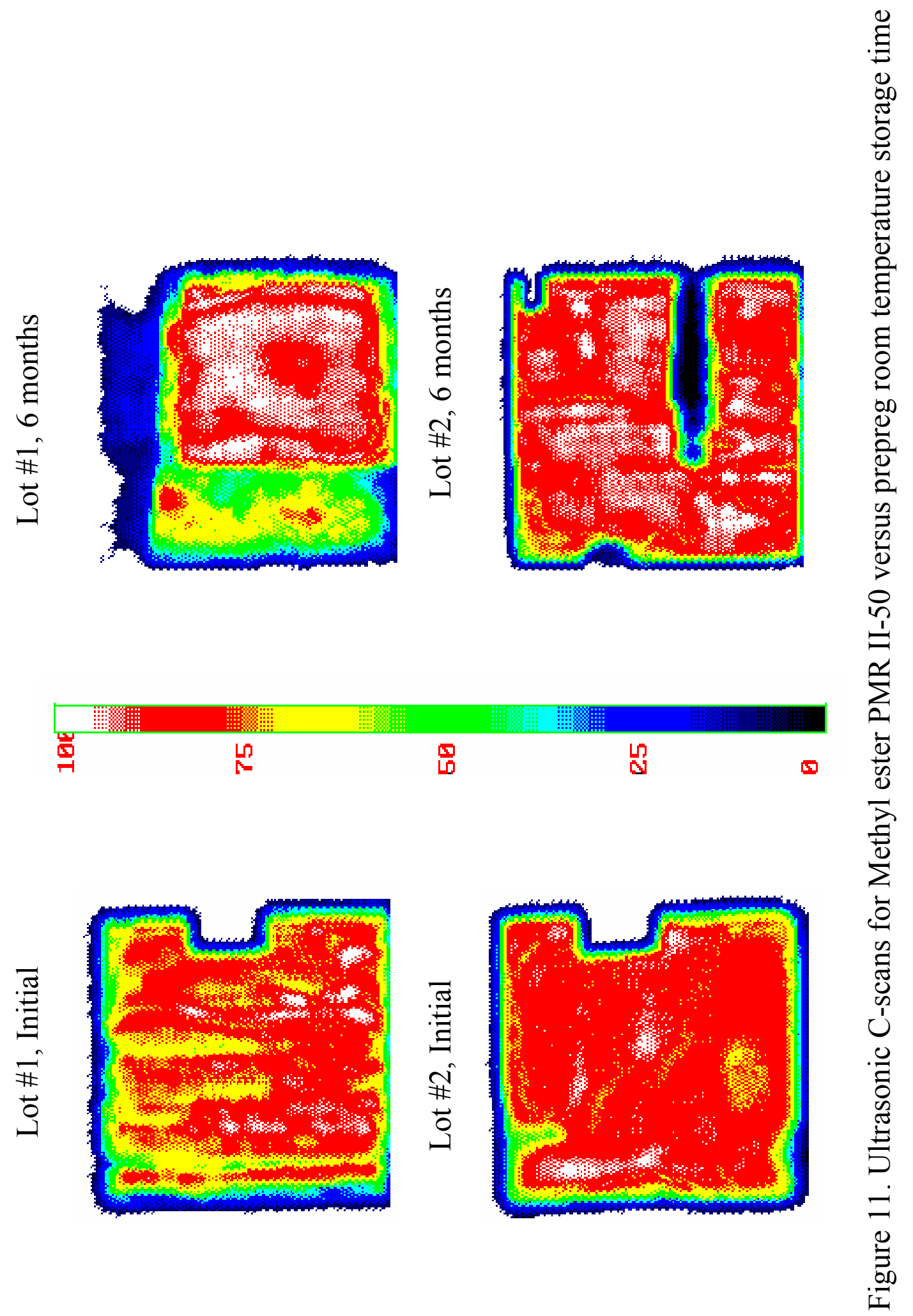



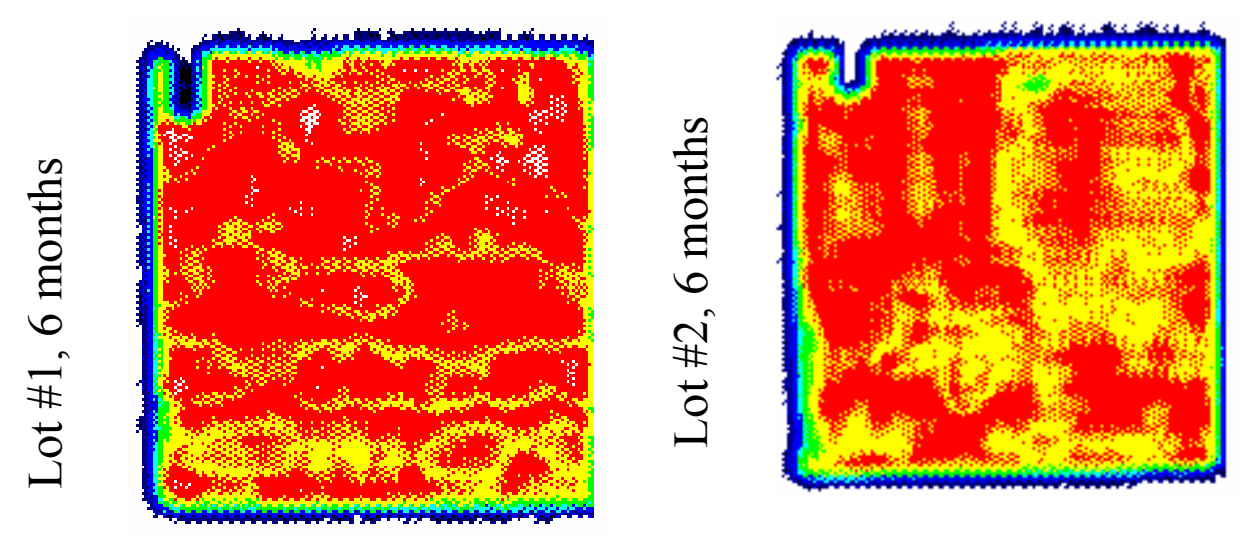

回

00

范

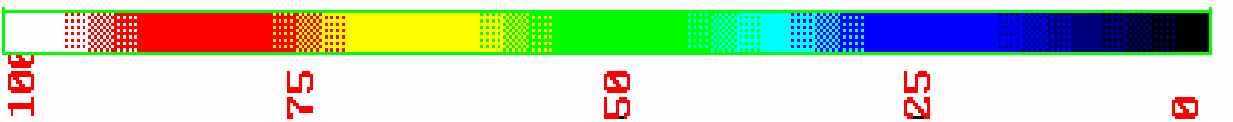

$\nabla$
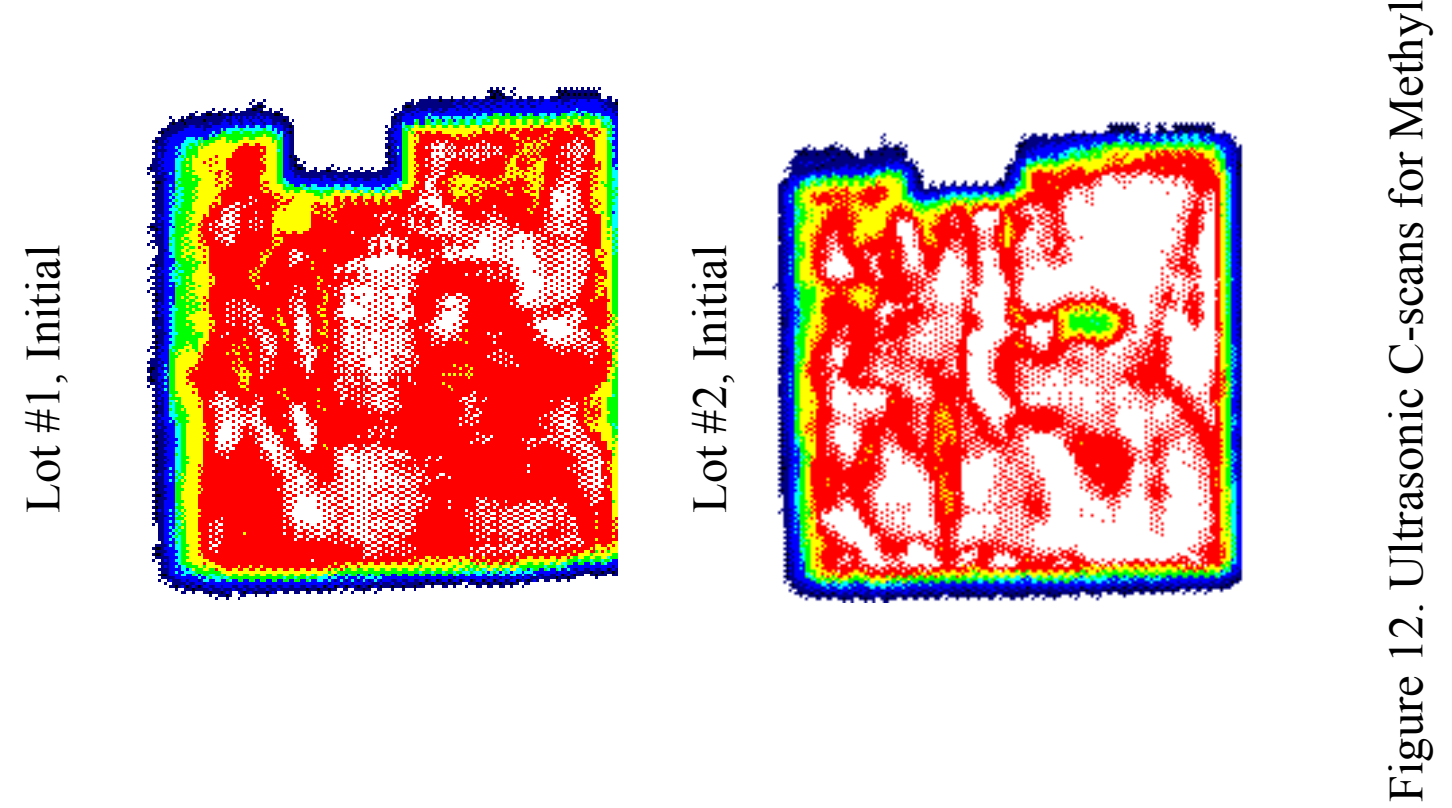

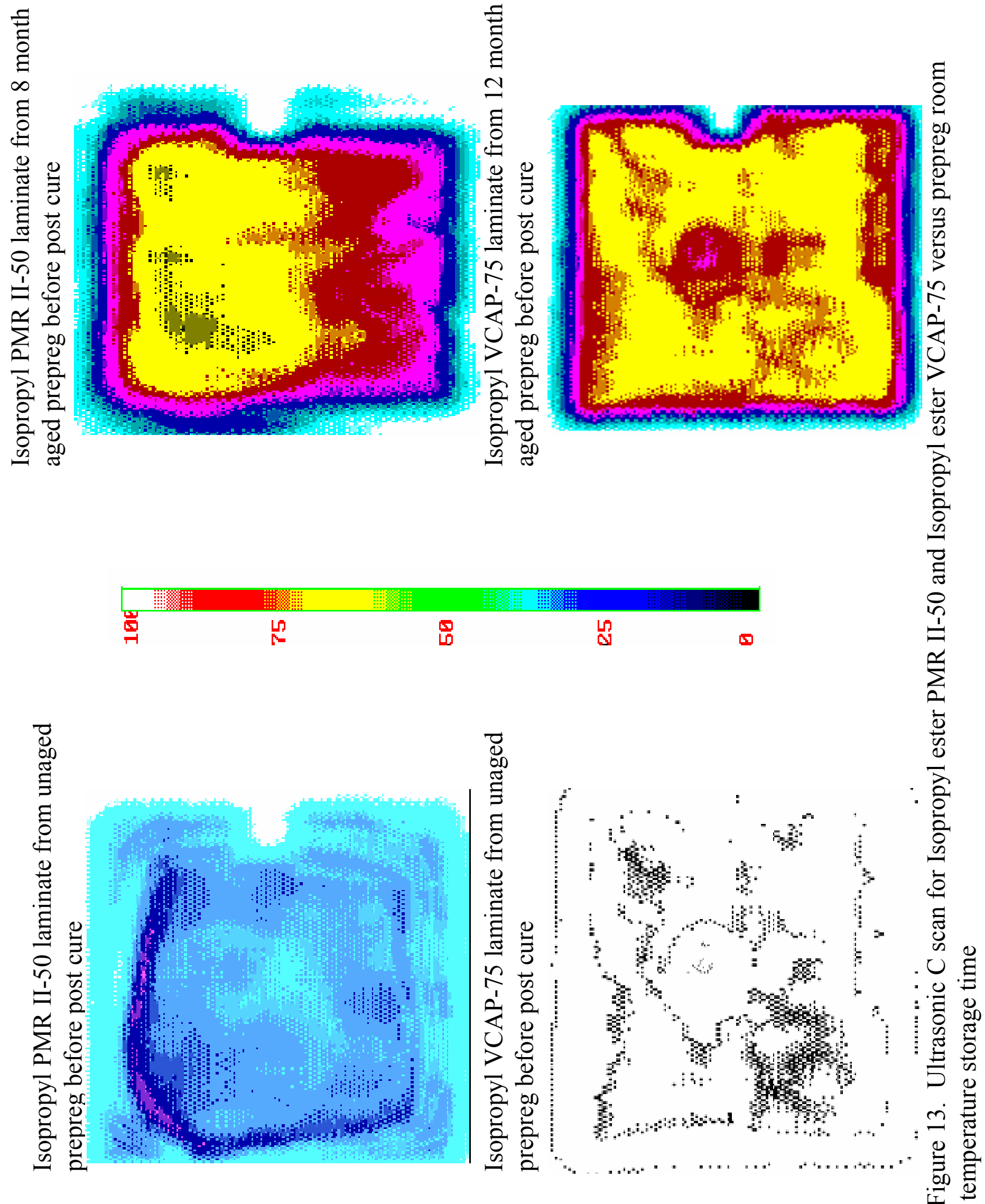


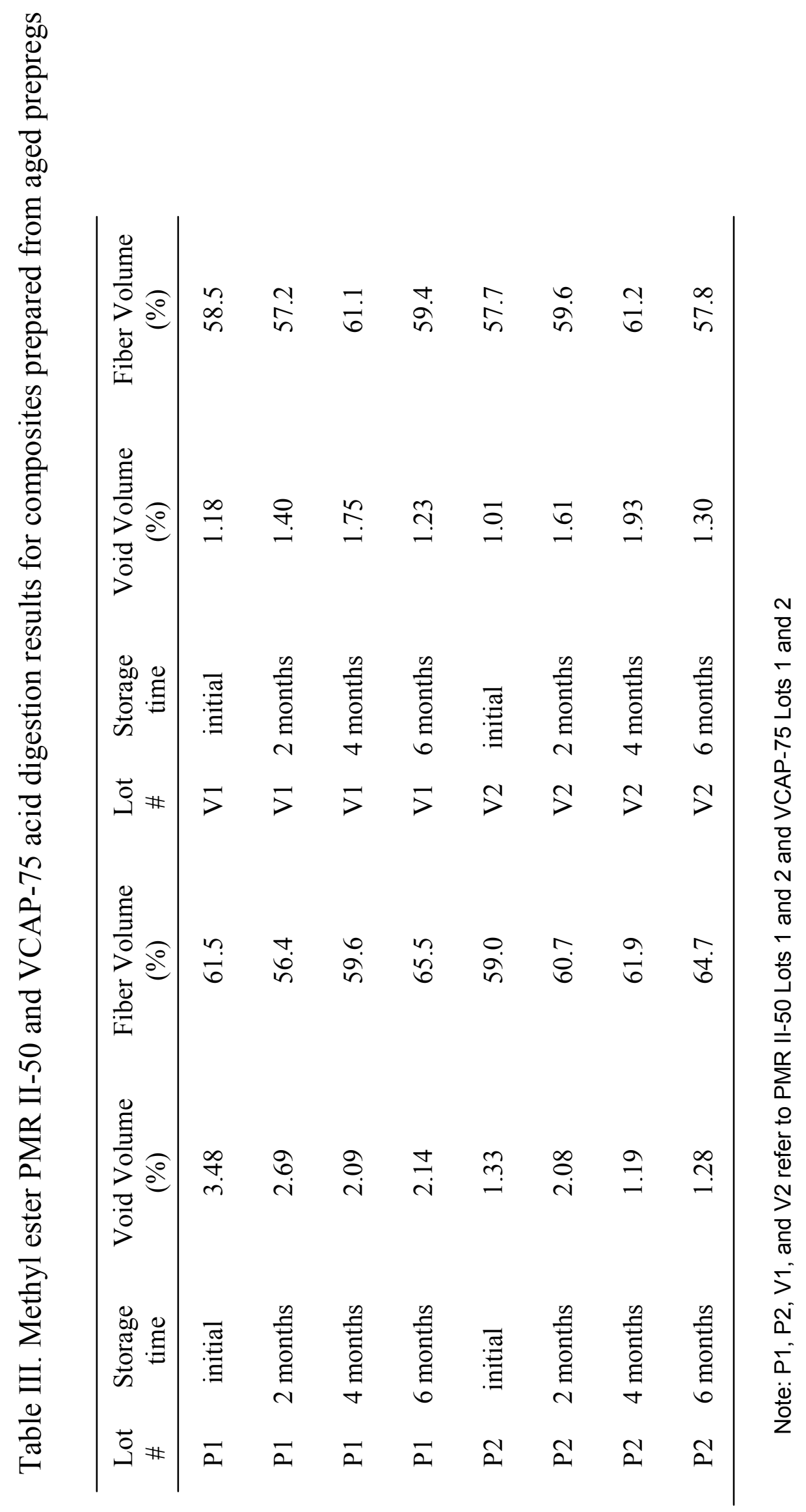




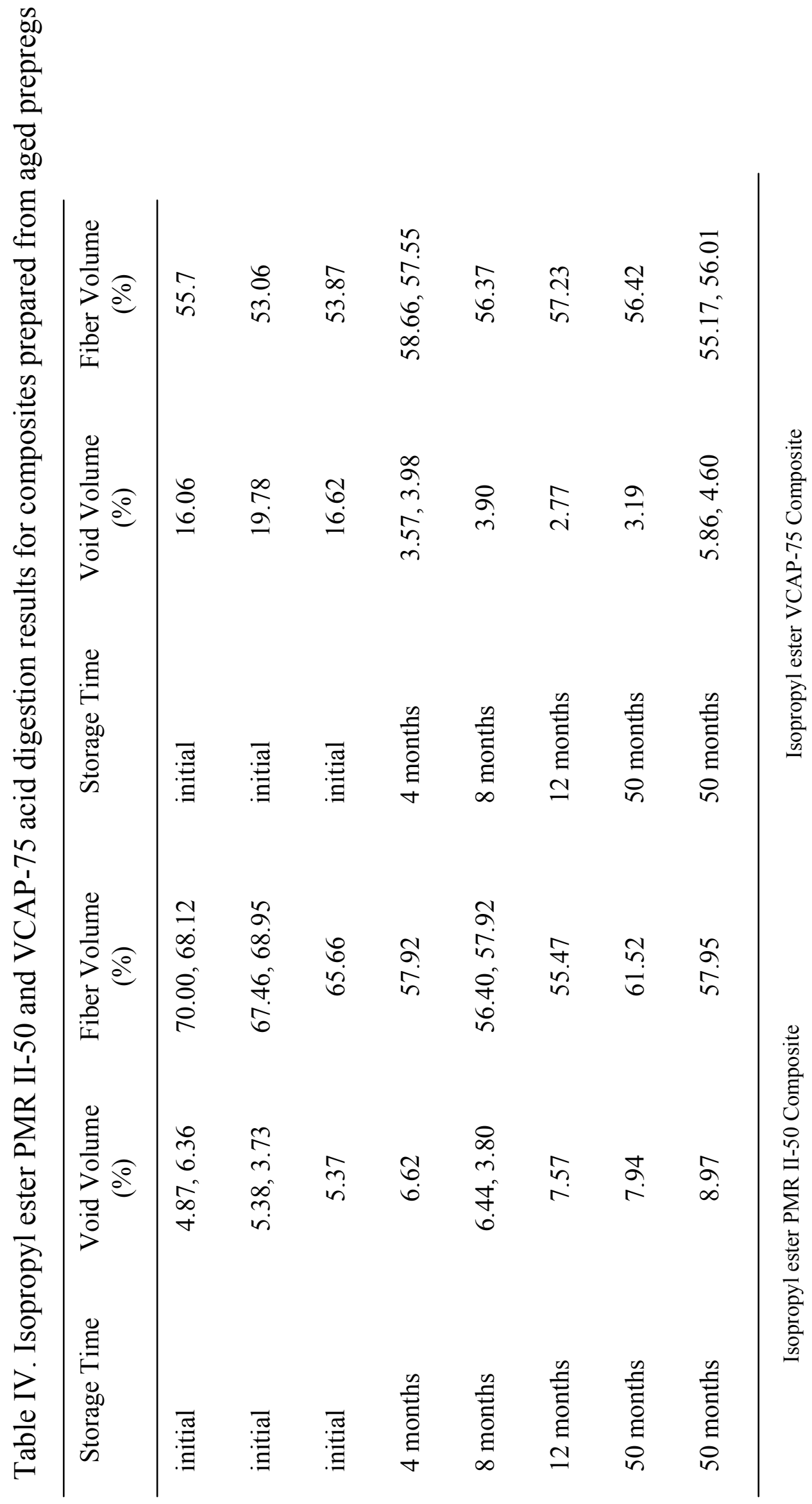




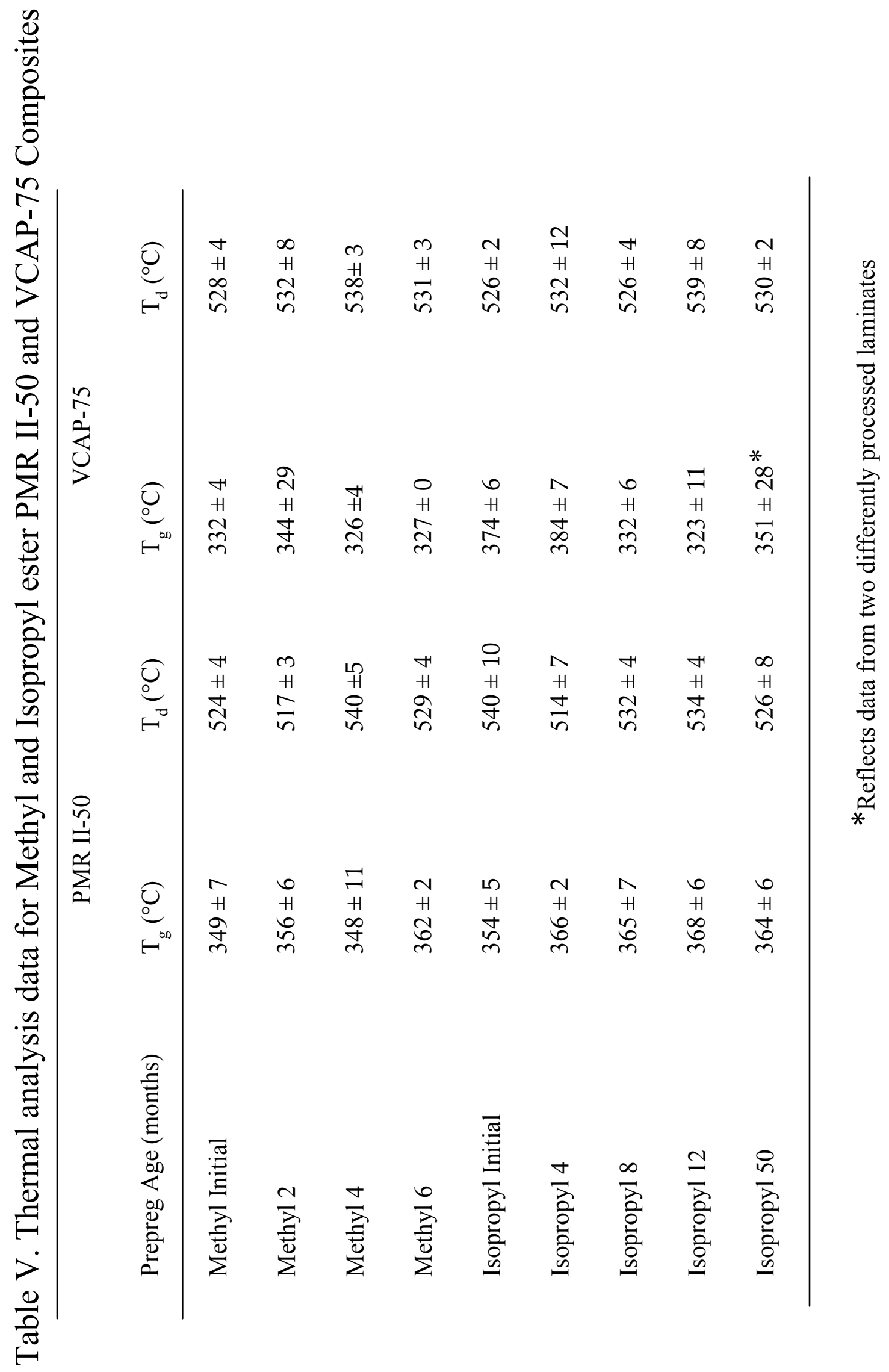




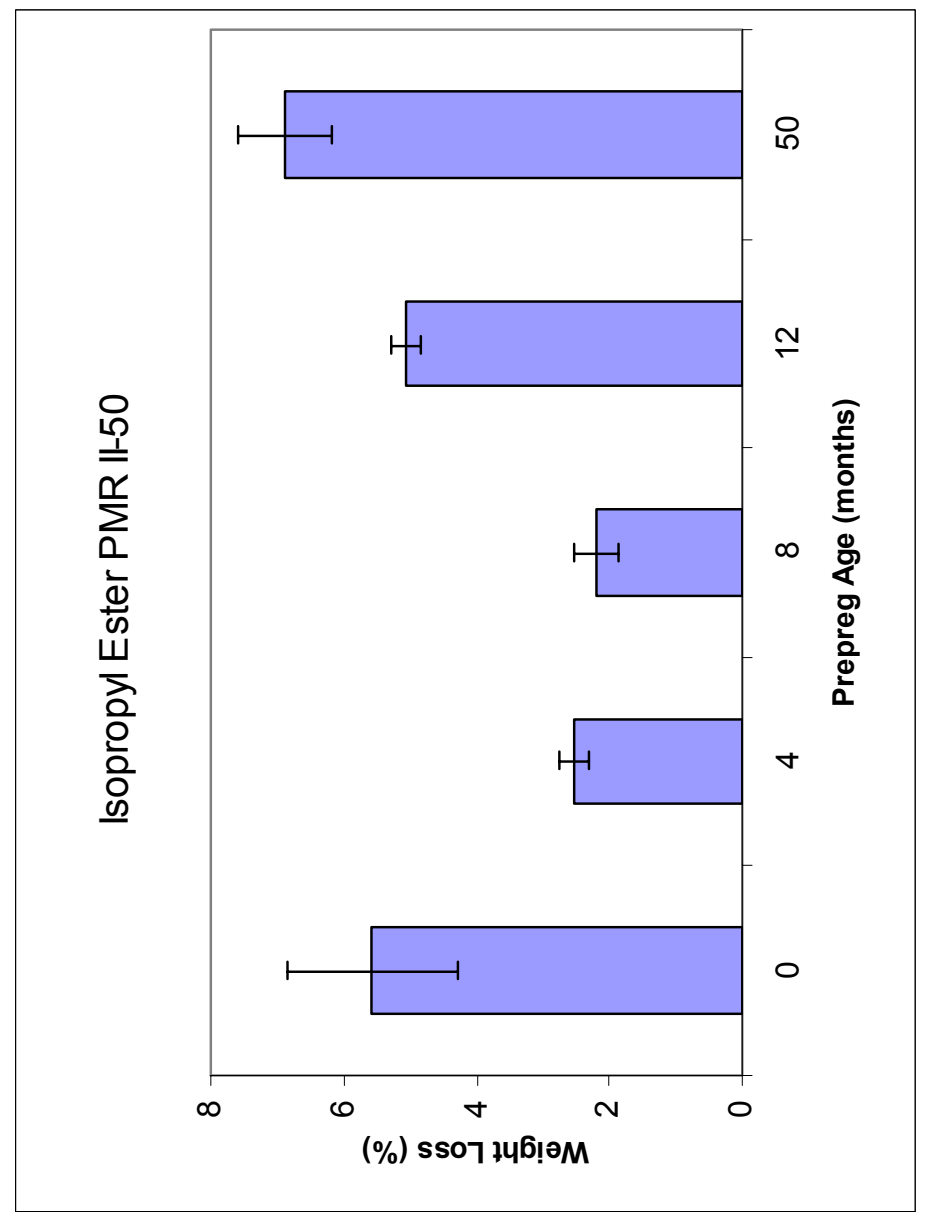

0
0
0
0
0
0
0
0
0
0
0
0
0
0
0
0
0
0
0
0
0
0
0
0
0
0
0
0
0
0
0
0

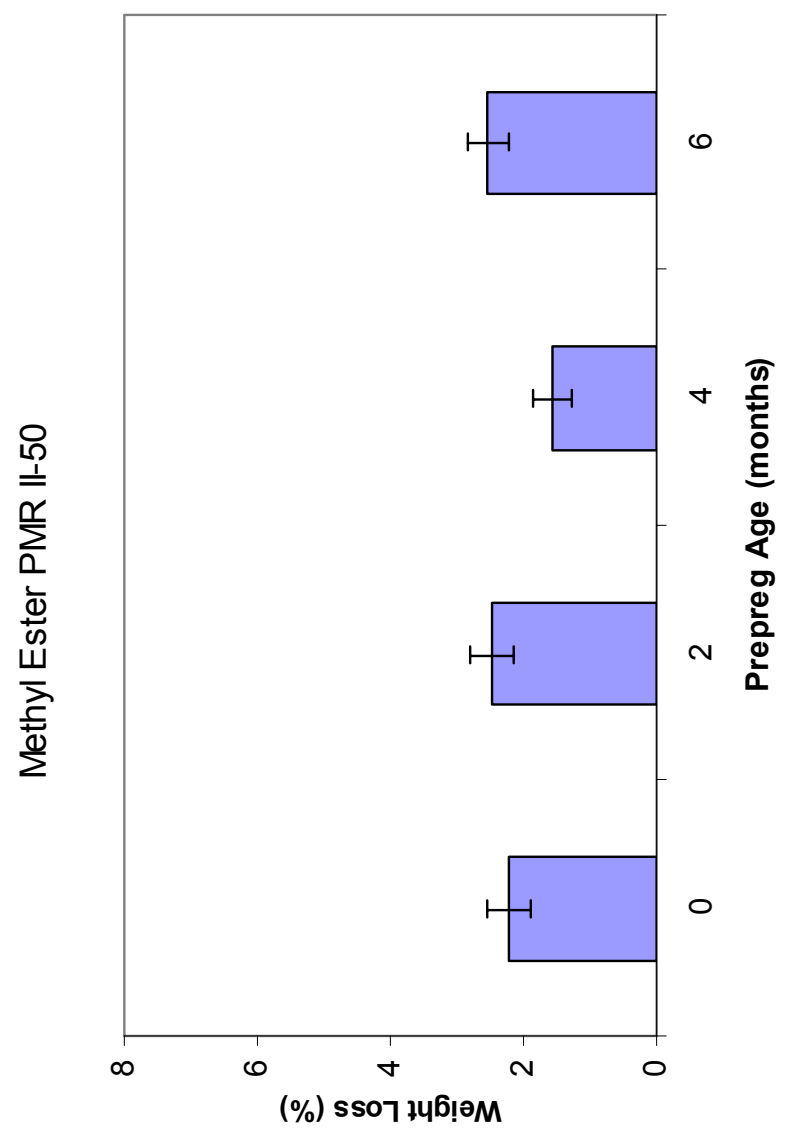

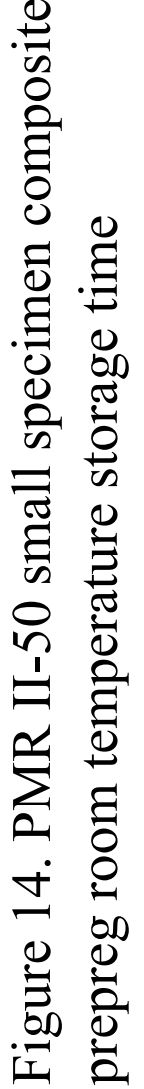




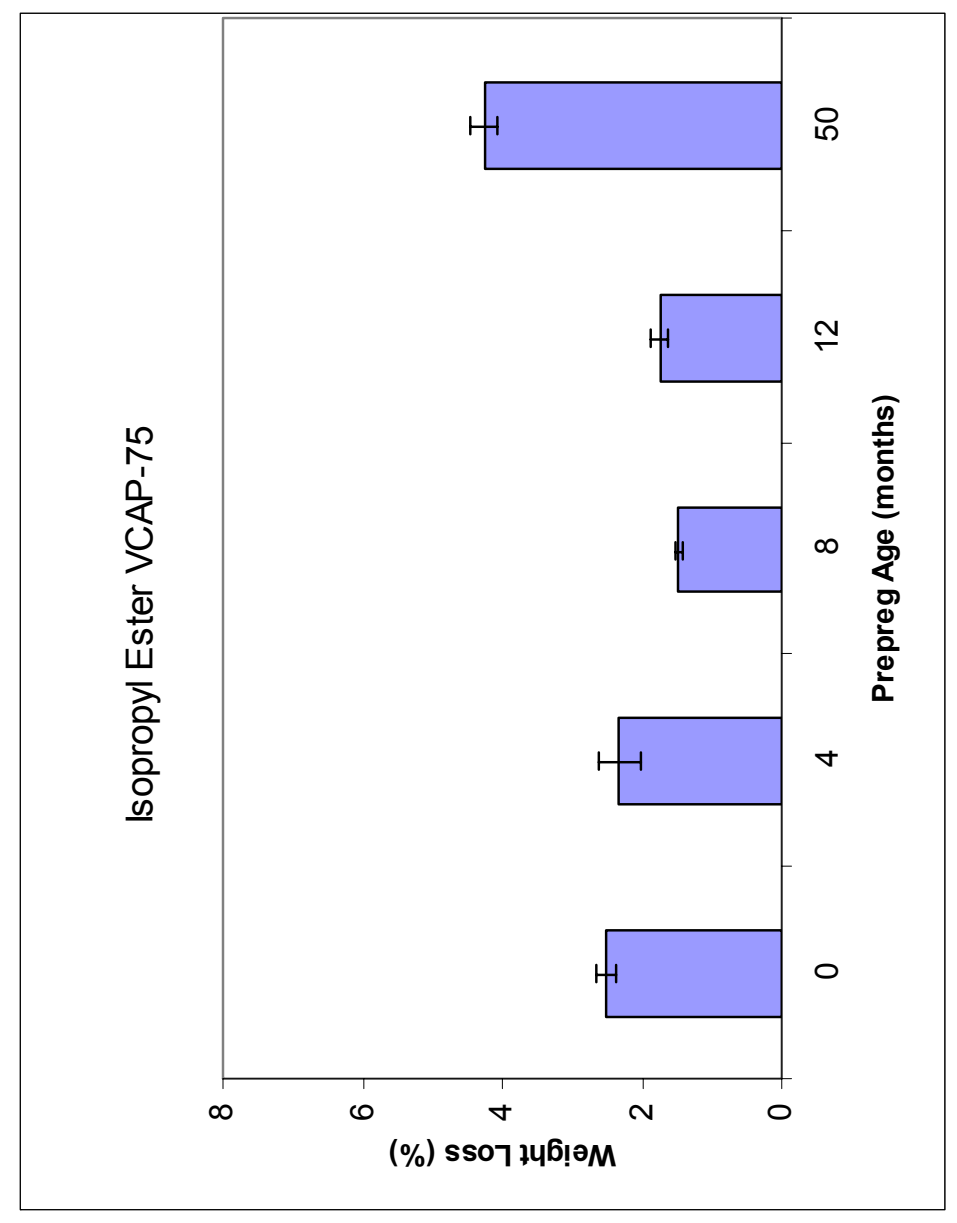

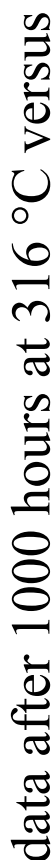

\&

क्ञ

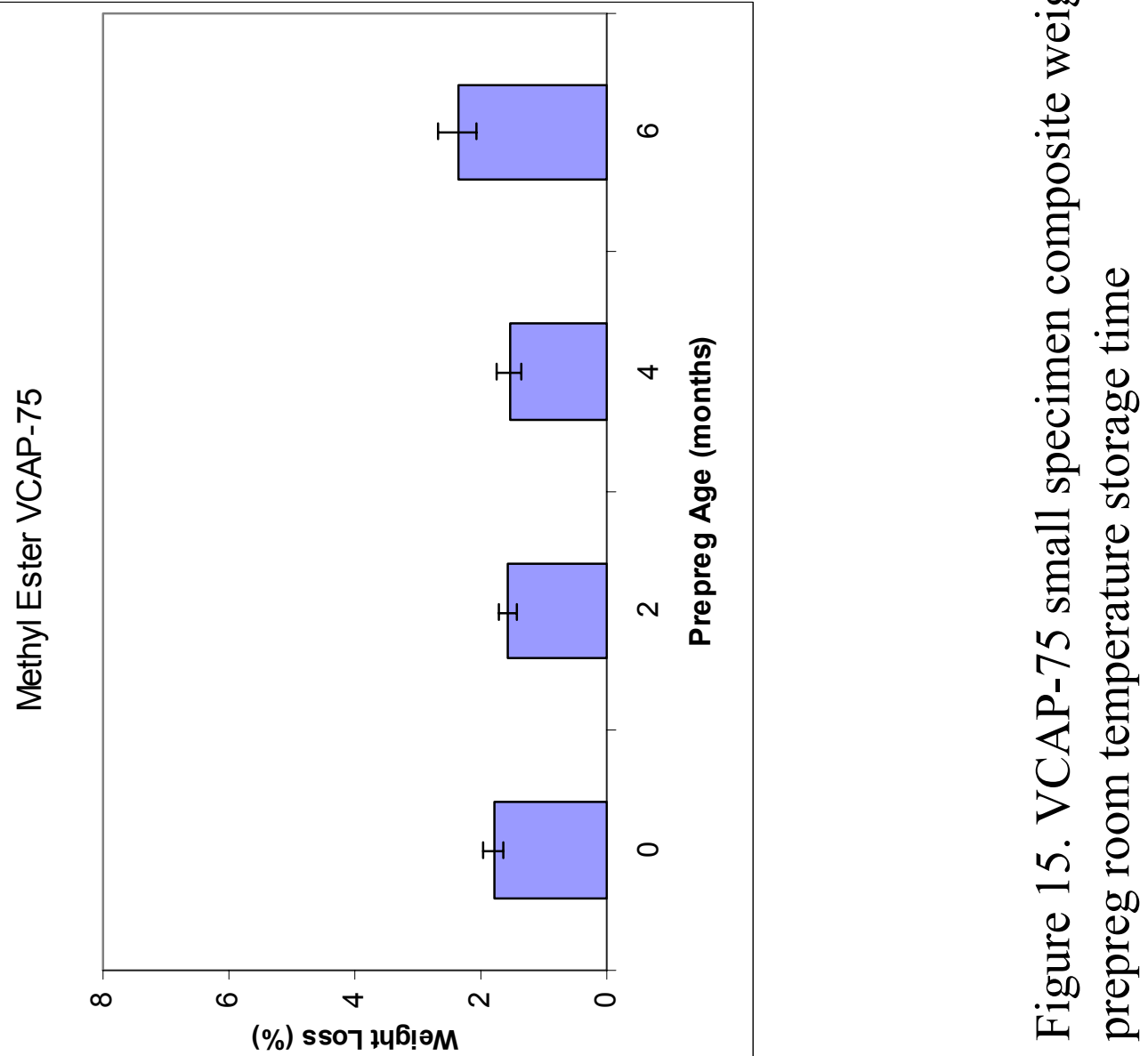




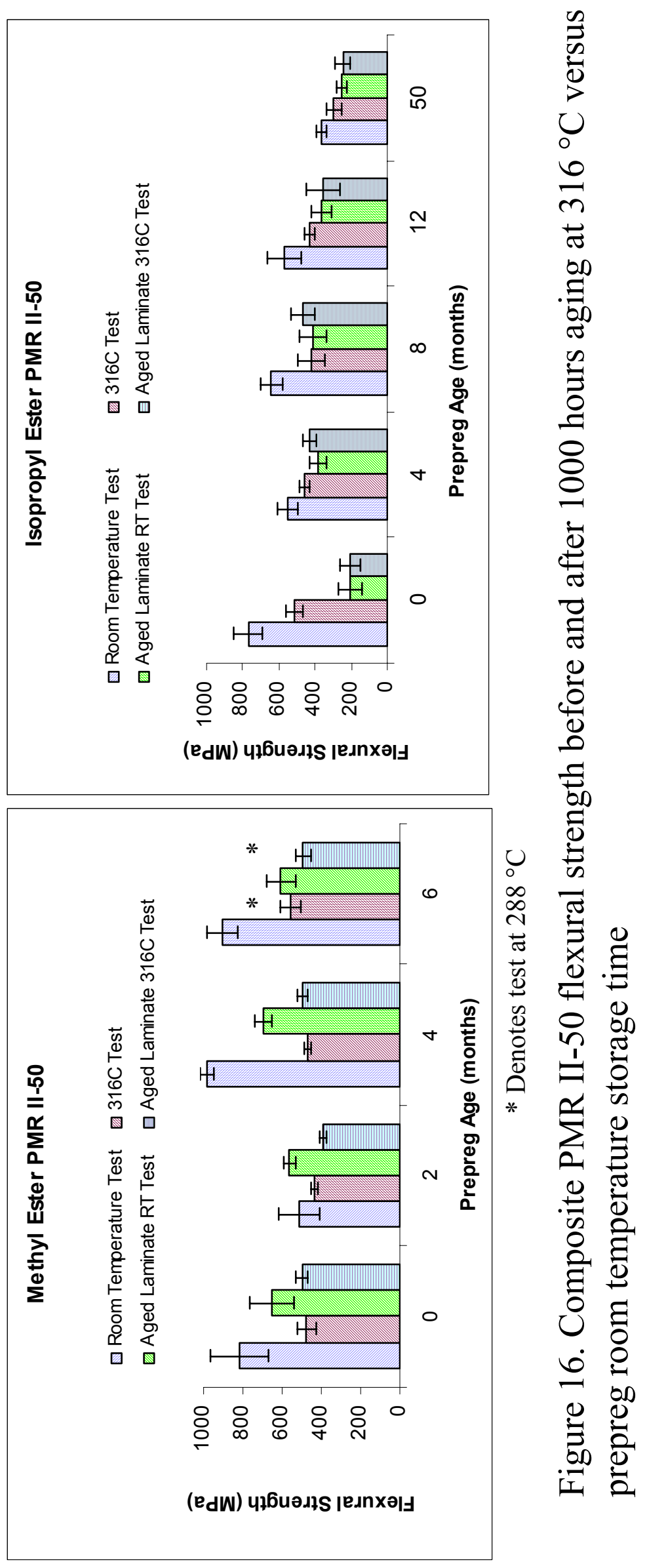




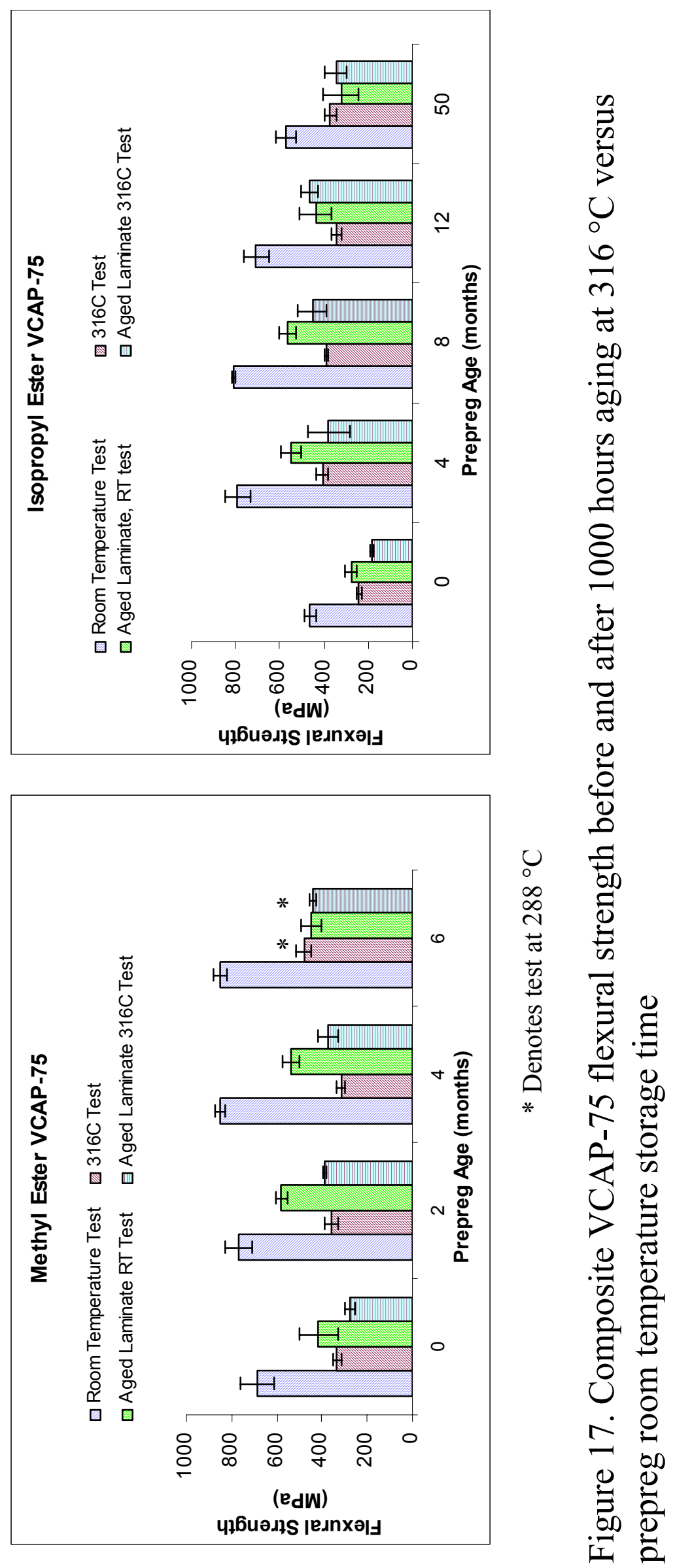



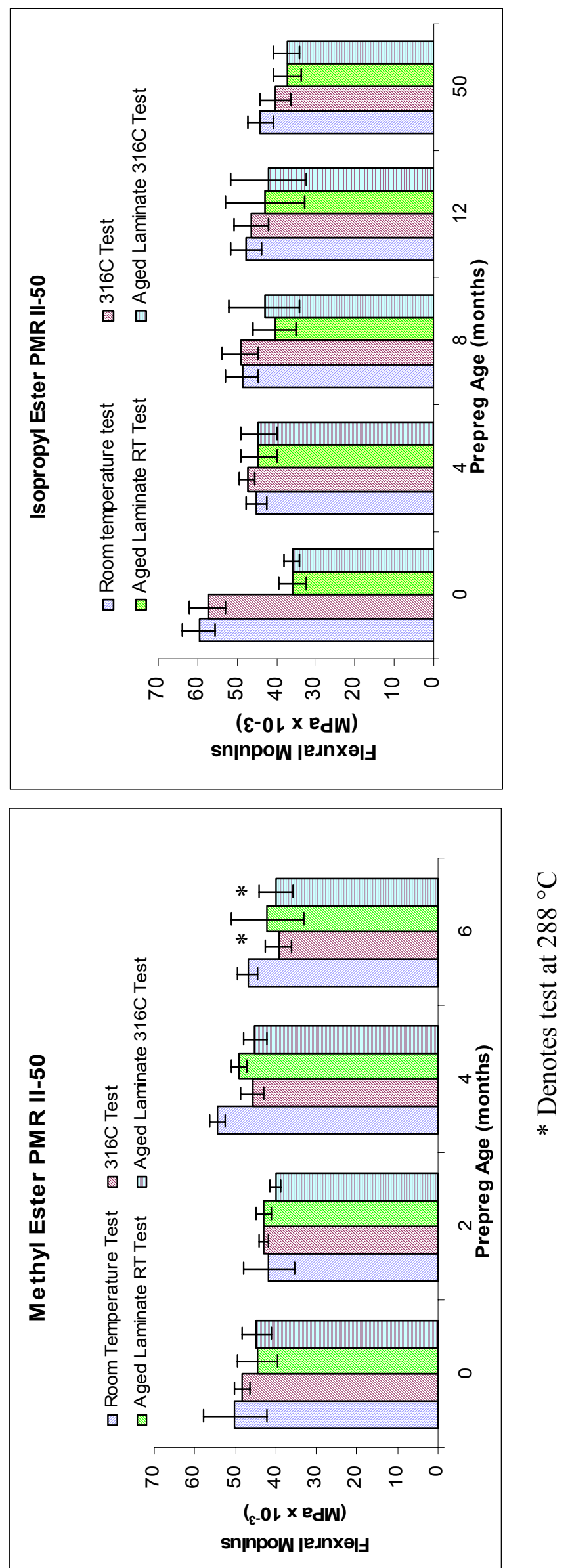


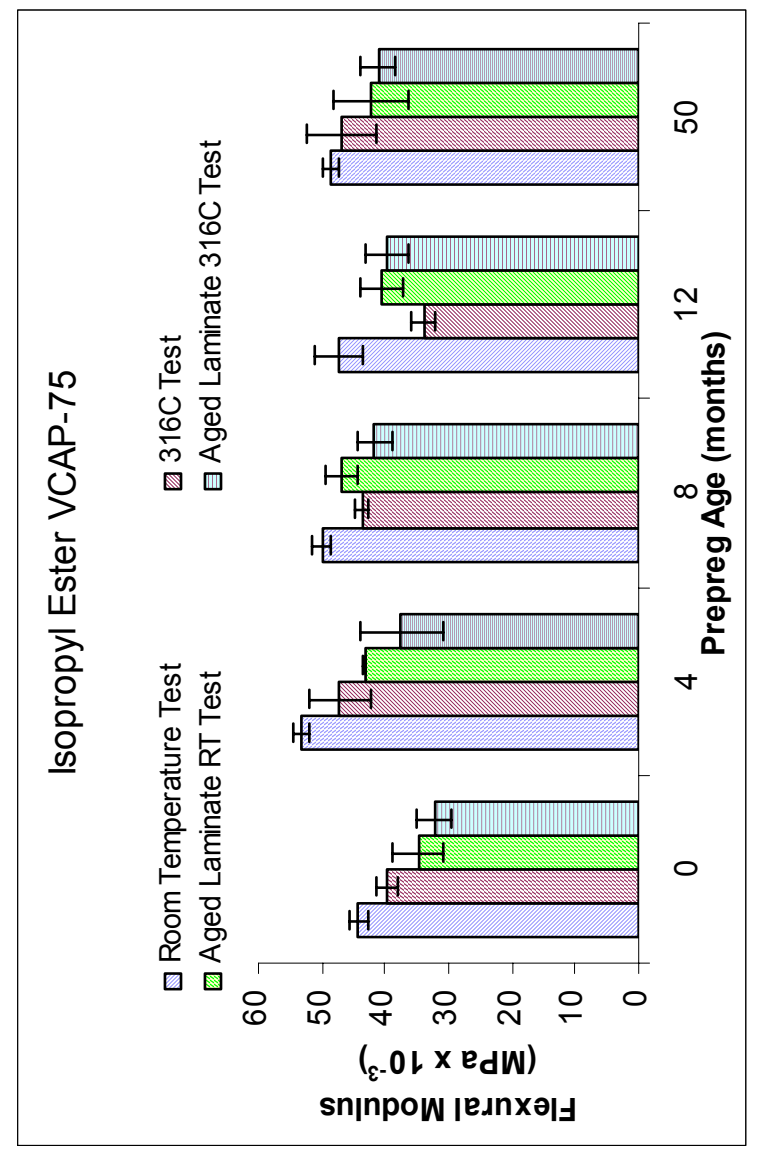

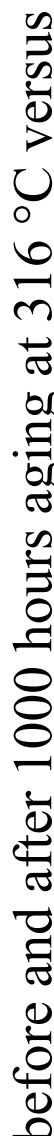
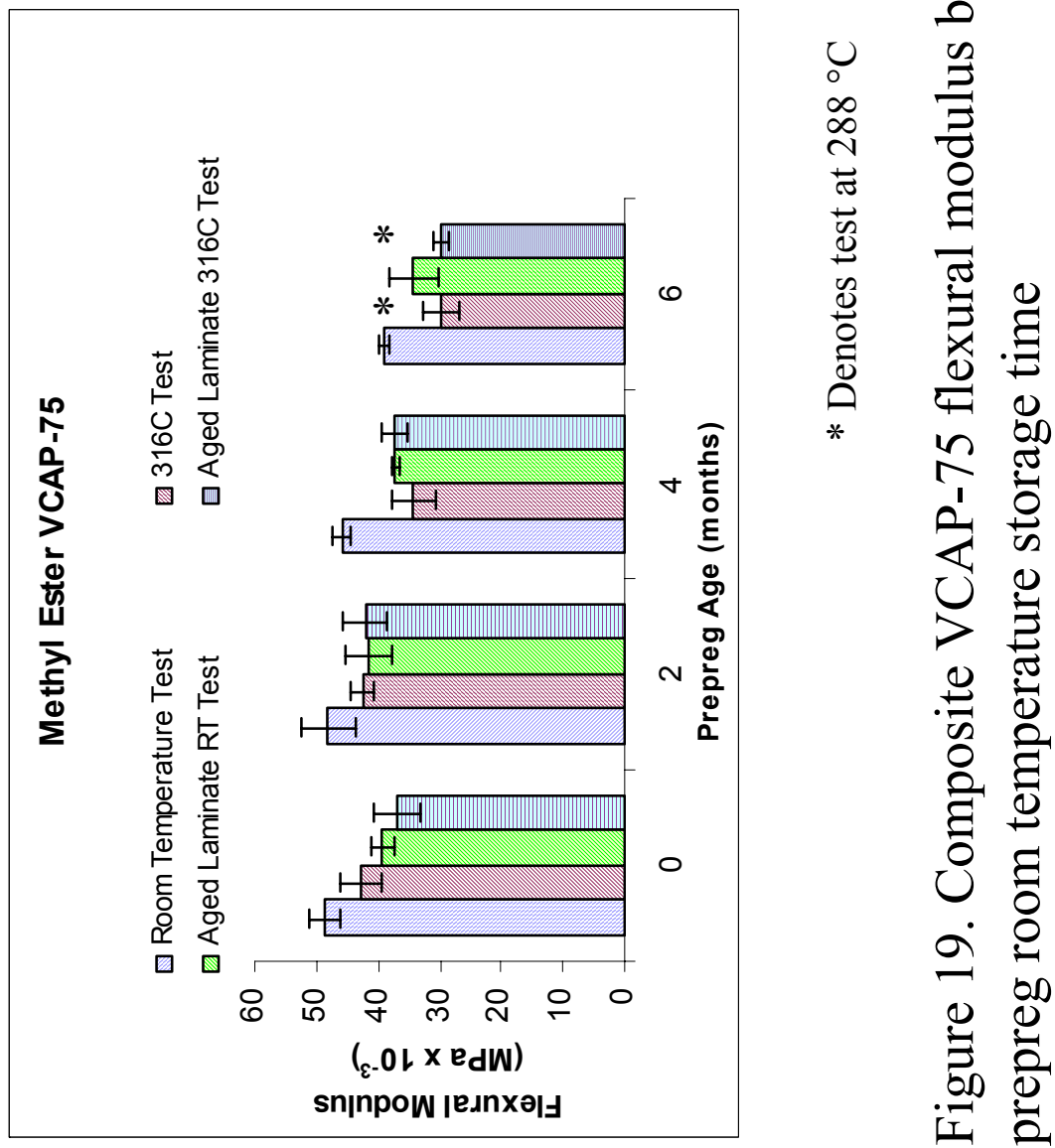